University of Nebraska - Lincoln

DigitalCommons@University of Nebraska - Lincoln

$10-1-2006$

\title{
Homogeneous nucleation at high supersaturation and heterogeneous nucleation on microscopic wettable particles: A hybrid thermodynamic/density-functional theory
}

\author{
T. V. Bykov \\ McMurry University \\ Xiao Cheng Zeng \\ University of Nebraska-Lincoln, xzeng1@unl.edu
}

Follow this and additional works at: https://digitalcommons.unl.edu/chemzeng

Part of the Chemistry Commons

Bykov, T. V. and Zeng, Xiao Cheng, "Homogeneous nucleation at high supersaturation and heterogeneous nucleation on microscopic wettable particles: A hybrid thermodynamic/density-functional theory" (2006). Xiao Cheng Zeng Publications. 3.

https://digitalcommons.unl.edu/chemzeng/3

This Article is brought to you for free and open access by the Published Research - Department of Chemistry at DigitalCommons@University of Nebraska - Lincoln. It has been accepted for inclusion in Xiao Cheng Zeng Publications by an authorized administrator of DigitalCommons@University of Nebraska - Lincoln. 


\title{
Homogeneous nucleation at high supersaturation and heterogeneous nucleation on microscopic wettable particles: A hybrid thermodynamic/density-functional theory
}

\author{
T. V. Bykov and X. C. Zeng \\ Department of Physics, McMurry University, Abilene, Texas 79697 and Department of Chemistry, \\ University of Nebraska-Lincoln, Lincoln, Nebraska 68588
}

(Received 7 July 2006; accepted 1 September 2006; published online 11 October 2006)

\begin{abstract}
Homogeneous nucleation at high supersaturation of vapor and heterogeneous nucleation on microscopic wettable particles are studied on the basis of Lennard-Jones model system. A hybrid classical thermodynamics and density-functional theory (DFT) approach is undertaken to treat the nucleation problems. Local-density approximation and weighted-density approximation are employed within the framework of DFT. Special attention is given to the disjoining pressure of small liquid droplets, which is dependent on the thickness of wetting film and radius of the wettable particle. Different contributions to the disjoining pressure are examined using both analytical estimations and numerical DFT calculation. It is shown that van der Waals interaction results in negative contribution to the disjoining pressure. The presence of wettable particles results in positive contribution to the disjoining pressure, which plays the key role in the heterogeneous nucleation. Several definitions of the surface tension of liquid droplets are discussed. Curvature dependence of the surface tension of small liquid droplets is computed. The important characteristics of nucleation, including the formation free energy of the droplet and nucleation barrier height, are obtained. (C) 2006 American Institute of Physics. [DOI: 10.1063/1.2357937]
\end{abstract}

\section{INTRODUCTION}

This paper is closely related to an earlier work by us, ${ }^{1}$ in which we considered heterogeneous nucleation on mesoscopic wettable particles. The theoretical method developed there was a hybrid thermodynamics/density-functional theory approach within the weighted-density approximation. It is well known that heterogeneous nucleation is the most common mechanism to initiate a first-order phase transition from metastable supersaturated vapor to bulk liquid. With wettable particles the nucleation proceeds through formation of a liquidlike film on the surface of the particles. Although a variety of particles can act as heterogeneous nucleation centers, in Ref. 1 we only considered mesoscopic wettable particles as a possible source to initiate heterogeneous nucleation. In this work we extend the approach proposed in the earlier study to deal with microscopic wettable particles. In addition, we studied homogeneous nucleation as a limiting case of heterogeneous nucleation as the size of wettable particles approaches to zero.

In Ref. 1 we showed that the behavior of disjoining pressure and chemical potential of liquid films formed on the surface of mesoscopic wettable particles is responsible for the heterogeneous nucleation mechanism. One of the key characteristics of heterogeneous nucleation is the existence of a threshold supersaturation. If the particle was placed in a supersaturated vapor with supersaturation higher than this threshold value then the barrierless nucleation would occur. This is why the initiation of homogeneous nucleation always requires higher value of supersaturation than that for heterogeneous nucleation.

Note that even homogeneous nucleation can become bar- rierless if the chemical potential of the supersaturated vapor exceeds the spinodal value. However, the classical nucleation theory ${ }^{2}$ cannot predict the threshold chemical potential in the case of homogeneous nucleation. Several attempts have been made recently to modify this theory in order to account for the existence of the threshold. For instance, the scaling relations for critical nucleus were introduced by McGraw and Laaksonen $^{3}$ and further tested by Talanquer. ${ }^{4}$ The McGrawLaaksonen phenomenological theory gives significant improvement over the classical nucleation theory even though there is still a tendency to overestimate the nucleation barrier at high supersaturation and underestimate the height of nucleation barrier at low supersaturation. The scaling law found further support in thermodynamically and kinetically consistent extended modified liquid drop (EMLD) model, proposed by Reguera and Reiss. ${ }^{5}$ This theory gives even better agreement with simulation/experimental results since EMLD takes into account fluctuations existing in extremely small clusters. Thermodynamically this means that small homogeneous systems are already heterogeneous and additional contributions have to be introduced into thermodynamic potential to study these systems. Another purely thermodynamic approach has been pursued by Kashchiev, ${ }^{6}$ in which a phenomenological approximation has been made to account for the existence of the spinodal. We shall use the idea of nonhomogeneity of small droplets and explore some physical reasons behind aforementioned phenomenological approximations.

The similarity in heterogeneous and homogeneous nucleation mechanisms becomes closer as the size of the impurity particle becomes smaller. When the impurity par- 
ticle and the molecules of the nucleating substance are about the same size, one could view the nucleation as a binary nucleation rather than heterogeneous nucleation. Here we only consider very dilute concentration of impurity particles and that the size of the particle is a few times larger than that of molecules of nucleating substance. As such, we still can treat the nucleation process as heterogeneous nucleation. We also used the assumption made in Ref. 1, that is, the droplet formation proceeds via forming a thin fluid film on the surface of the impurity particle ("complete wetting"). The thin film then grows into a larger spherical droplet with the impurity particle located at the center.

Thermodynamic theory of heterogeneous nucleation ${ }^{7-9}$ as well as our previous theoretical work ${ }^{1}$ are all based on the perturbation approach with an expansion over small curvature of the liquid-vapor interface. The classical theory ${ }^{7-9}$ used a zero-order approximation which is only valid for macroscopic nucleation centers. The theory in Ref. 1 involves the first-order approximation which includes the Tolman curvature correction and compressibility correction. The first-order approximation allows us to deal with mesoscopic wettable particles. However, for microscopic wettable particles the perturbation approach is no longer effective. The reasons are given below.

First, the surface tension of a very small droplet is very different from that in the planar limit. For large droplets the Tolman correction to the surface tension can be used in the planar limit. ${ }^{1}$ In fact, the value of the Tolman length is extremely small, possibly even close to zero. ${ }^{1,10,11}$ However, the first-order curvature correction cannot be used for microscopic droplets, since the Tolman length itself is highly dependent on the curvature ${ }^{12}$ while the surface tension of small droplets (in conventional thermodynamic sense) is close to zero. $^{13-15}$

Second, the fluid density of small droplet is no longer close to bulk liquid density (in coexistence with saturated vapor). Moreover, this density cannot be approximated using compressibility correction. While the compressibility route predicts the bulk density to be larger than that in the planar limit, the fluid density of the small droplet approaches to vapor value $\mathrm{v}^{14-16}$ as supersaturation increases. The EMLD model $^{5}$ also supports that at high supersaturations the critical cluster is a diffusive physical entity containing a significant number of vaporlike molecules. This is analog to the behavior of fluid density of a thin liquid film on the solid substrate, where surface layers overlap. ${ }^{1,7}$ To describe this behavior, the disjoining pressure of a thin liquid film has to be considered. Similarly, the disjoining pressure should be introduced when considering very small droplets, due to the small thickness of the film on the surface of the impurity particles, as well as the small size of the droplet. We expect similar effect in homogeneous nucleation as well. . $^{14,15,17}$

We employed classical density-functional theory (DFT) as a theoretical tool to calculate thermodynamic characteristics of liquid condensate as in previous study. ${ }^{1}$ It is known that DFT can be used to investigate behavior of fluid near planar solid surface. ${ }^{18-22}$ We also used the weighted-density approximation (WDA) developed by Tarazona, ${ }^{18}$ and Tarazona et al. ${ }^{19}$ which has been proven to be effective to de- scribe highly nonuniform solid-liquid interfacial systems. Here we used the same DFT/WDA approach but for a spherical droplet system rather than for the planar system. The local-density approximation (LDA), although cannot qualitatively describe the density profile of fluid near the solid surface, is very effective to deal with homogeneous nucleation. ${ }^{12,14,23}$ However, to our knowledge, WDA has not been applied to deal with homogeneous nucleation. Using the DFT/WDA approach in spherical coordinates, not only we can treat the problem of heterogeneous nucleation on microscopic particles but also we can compare DFT/WDA with DFT/LDA in calculating quantities relevant to the homogeneous nucleation. For both types of nucleation, as in Ref. 1, we calculated dependence of the chemical potential of the liquid condensate on the droplet size, the threshold value of the chemical potential (or the threshold value of supersaturation) at which barrierless nucleation takes place, the height of the activation barrier, the location of minimum and maximum on the curve of free energy of droplet formation versus the droplet size, and a few other characteristics necessary to describe kinetics of nucleation.

This paper is organized as follows. In Sec. II thermodynamics of heterogeneous and homogeneous nucleations is discussed. The disjoining pressure is described in Sec. III. In Sec. IV the DFT/WDA approach is detailed to treat a spherical system. Characteristics of homogeneous nucleation at high supersaturation of vapor are obtained in Sec. V. Key properties of heterogeneous nucleation on microscopic particles are presented and discussed in Sec. VI. Discussions and conclusions are given in Sec. VII.

\section{THERMODYNAMICS OF NUCLEATION}

\section{A. Heterogeneous nucleation}

In the previous work ${ }^{1}$ we showed several thermodynamic equations such as the Laplace equation and GibbsDuhem equation to describe heterogeneous nucleation on mesoscopic wettable particles. The general forms of these equations remain valid to describe heterogeneous nucleation on microscopic wettable particles.

We summarize some main results of Ref. 1 in this section. We consider an open system which consists of a wettable solid particle and a metastable vapor at given temperature and volume. Upon vapor condensation, a fluid film with a uniform thickness is formed on the surface of the particle. Assuming spherical symmetry, the density profile of the system is given by $\rho(r)$, where $r$ is measured from the center of the solid particle, which is also the center of the droplet. We denote metastable vapor as phase 1, the fluid film as phase 2, and the solid particle as phase 3 . To describe thermodynamic properties of this nonuniform system, we employed the Gibbs method of dividing surfaces to replace the real density profile $\rho(r)$ by a step-function density profile. This stepfunction density profile consists of several regions. (1) A uniform region of solid particle with density $\rho_{3}$ for $r<\widetilde{R}_{n}$, where $\widetilde{R}_{n}$ is the physical radius of the solid particle. (2) A uniform fluid film with density $\rho_{2}$ which is equal to the bulk density of liquid at the same supersaturation for $R_{n}<r<R$, where $R_{n}$ is the radius of solid-liquid dividing surface. It 
differs from the radius of the particle $\widetilde{R}_{n}$ by $z_{0} \equiv R_{n}-\widetilde{R}_{n}$ (about few molecular diameters) which depends on the thickness of solid-liquid interface. The uniform density is set to be zero in a thin region $\widetilde{R}_{n}<r<R_{n}$. This reflects the fact that solid-liquid interaction potential is repulsive near the solid surface (this potential diverges at $r=\widetilde{R}_{n}$ ). (3) The uniform vapor with density of $\rho_{1}$ for $r>R$, where $R$ is the radius of liquid-vapor dividing surface. Thus, the thickness of the liquid film is given by $h=R-R_{n}$. Finally $L$ represents the outmost radius of the entire system and is chosen in such a way that beyond $L$ the physical density of vapor is nearly uniform $\left(=\rho_{1}\right)$. For the open system with long-range interactions $L$ $\rightarrow \infty$.

The supersaturation can be described by the chemical potential $\mu$. For given temperature this chemical potential also determines the density $\rho_{1}$ of bulk vapor and $\rho_{2}$ of bulk liquid, as well as the bulk vapor pressure $p_{1}$ and the bulk liquid pressure $p_{2}$. The grand thermodynamic potential of the system (excluding the solid particle) is given by

$$
\begin{aligned}
\Omega= & -p_{2} \frac{4 \pi}{3}\left(R^{3}-R_{n}^{3}\right)-p_{1} \frac{4 \pi}{3}\left(L^{3}-R^{3}\right)+4 \pi R_{n}^{2} \sigma_{32}\left(R_{n}\right) \\
& +4 \pi R^{2} \sigma_{21}(R)+\widetilde{\Omega}(h),
\end{aligned}
$$

where the notations are the same as used in Ref. 1: $\sigma_{32}\left(R_{n}\right)$ is the surface tension of the solid-liquid interface, which is dependent on the radius of dividing surface $R_{n}$; and $\sigma_{21}(R)$ is the surface tension of the liquid-vapor interface, which is dependent on the radius of the dividing surface $R$. Both $\sigma_{32}\left(R_{n}\right)$ and $\sigma_{21}(R)$ are defined in such a way that they are independent of the liquid film's thickness. The fact that thermodynamic potential depends on the finite thickness of the film $h$ is accounted for with the last term in Eq. (1), $\widetilde{\Omega}(h)$, which approaches to zero as $h$ approaches infinity. This last term can be expressed by

$$
\widetilde{\Omega}(h)=4 \pi \int_{R_{n}+h}^{L} r^{2} \widetilde{\omega}(r) d r,
$$

where $\widetilde{\omega}(r)=\left.\Pi\left(R_{n}, h\right)\right|_{h=r-R_{n}}$ is Derjaguin's disjoining pressure of thin fluid film.

Based on Eq. (1) we derived ${ }^{1}$ the generalized Laplace equation which is valid for both equilibrium and critical clusters,

$$
p_{2}-p_{1}=\frac{2 \sigma_{21}(R)}{R}+\frac{\partial \sigma_{21}}{\partial R}(R)-\widetilde{\omega}\left(R_{n}+h\right) .
$$

Up to this point we have not made any assumptions about the size of the impurity particle covered by the droplet. So the Laplace equation (3) is also valid for microscopic nucleation center as for mesoscopic nucleation center. Moreover Eq. (3) is valid for homogeneous nucleation too. But for each of these cases we need to specify the nature of the last term $\widetilde{\omega}\left(R_{n}+h\right)=\widetilde{\omega}(R)=\Pi\left(R_{n}, h\right)$, which is given below.

Another important thermodynamic equation is the generalized Gibbs-Duhem equation, which can be derived based on Eq. (1) also. In the case of heterogeneous nucleation not one but two equations should be considered:

$$
d \sigma_{32}=-\Gamma_{32} d \mu
$$

and

$$
d \sigma_{21}-\frac{\partial \sigma_{21}}{\partial R} d R=-\Gamma_{21} d \mu,
$$

where $\Gamma_{32}=N_{32} / 4 \pi R_{n}^{2}$ is the adsorption of liquid on the surface of the solid particle, and $\Gamma_{21}=N_{21} / 4 \pi R^{2}$ is the adsorption at the liquid-vapor interface. The generalized Laplace equation (3) and Gibbs-Duhem equations (4) and (5) are the key thermodynamic equations required to study heterogeneous nucleation.

\section{B. Homogeneous nucleation}

To describe homogeneous nucleation, we set $R_{n}=0$ so that Eq. (1) becomes

$$
\Omega=-p_{2} \frac{4 \pi}{3} R^{3}-p_{1} \frac{4 \pi}{3}\left(L^{3}-R^{3}\right)+4 \pi R^{2} \sigma_{21}(R)+\widetilde{\Omega}(R) .
$$

The last term is now given by

$$
\widetilde{\Omega}(R)=4 \pi \int_{R}^{L} r^{2} \widetilde{\omega}(r) d r,
$$

because the grand thermodynamic potential is dependent on the finite size of the droplet. We can still write $\widetilde{\omega}(R)$ $=\Pi(0, R)=\Pi(R)$ as a definition of disjoining pressure. As such, the Laplace equation (3) for the critical cluster now becomes

$$
p_{2}-p_{1}=\frac{2 \sigma_{21}(R)}{R}+\frac{\partial \sigma_{21}(R)}{\partial R}-\Pi(R) .
$$

According to the definition of Derjaguin et al. ${ }^{24}$ the disjoining pressure is the difference between the normal component of the pressure tensor inside of the liquid film [i.e., at the center of droplet $p_{N}(0)$ here] and the pressure of bulk liquid $p_{2}$, given the same chemical potential $\mu$,

$$
\Pi=p_{N}(0)-p_{2} .
$$

Combining Eqs. (8) and (9) results in

$$
p_{N}(0)-p_{1}=\frac{2 \sigma_{21}(R)}{R}+\frac{\partial \sigma_{21}(R)}{\partial R},
$$

which is an alternative version of the Laplace equation compared to the conventional thermodynamic version often used in classical nucleation theory:

$$
p_{2}-p_{1}=\frac{2 \sigma_{21 t}(R)}{R}+\frac{\partial \sigma_{21 t}(R)}{\partial R},
$$

where the subscript $t$ denotes a different definition of thermodynamic surface tension.

The reason why Eqs. (10) and (11) are different is because the density inside a very small liquid droplet differs from that in bulk liquid. As a result, the normal component of pressure tensor inside of this small nonuniform droplet is different from the bulk liquid pressure. This effect is similar 
to the overlap of surface layer of thin liquid films, which can be accounted for by introducing the disjoining pressure in Eq. (8).

In our earlier works on homogeneous nucleation ${ }^{14,15}$ we used Eq. (11) as the Laplace equation. This conventional form cannot distinguish different physical contributions to thermodynamic potential. In contrast, Eq. (8) includes two terms. The curvature dependence of surface tension is solely due to the Tolman effect, which reflects the fact that liquidvapor interface has a finite thickness. Even without the overlap of the surface layer the change of the shape (curvature) of the interface should cause change in surface tension. This is a relatively weak effect due to small but finite compressibility of liquid, which is not included in classical capillary approximation. The last term (disjoining pressure) in Eq. (8) is due to interactions between molecules of liquid, which is not directly related to the compressibility. This contribution can be viewed as volume contribution rather than surface contribution, as traditionally treated in theory of nucleation. We will discuss a simple estimation for this term in the next section. The conventional form of the Laplace equation (11) attributes all curvature-related effects to the surface tension term. Finally, the Gibbs-Duhem equation (5) is still valid for homogeneous droplet, while Gibbs-Duhem equation (4) is not required because of the nonexistence of the solid-liquid interface.

\section{ANALYTICAL ESTIMATION OF THE DISJOINING PRESSURE}

The free energy density $\widetilde{\omega}(r)$ in Eq. (2) is also the disjoining pressure $\Pi\left(R_{n}, h\right)$. In the previous work ${ }^{1}$ we considered the wettable particles to be mesoscopic in size so that we can approximate the disjoining pressure by the corresponding planar value $\Pi(h)=\lim _{\tilde{R}_{n} \rightarrow \infty} \Pi\left(\widetilde{R}_{n}, h\right)$, assuming that the model solid-liquid interaction potential depends only on the distance from the surface of the solid. We showed in Ref. 1 that the power-law approximation for disjoining pressure is valid when the liquid film is thicker than about five molecular diameters, in which the overlapping effect is not very strong so that the center of the film can be treated as bulklike phase. Meanwhile, small changes in liquid density due to compressibility are almost compensated by weak overlapping. In that case van der Waals interactions play a main role and

$$
\Pi(h) \approx \frac{B}{\left(z_{0}+h\right)^{3}},
$$

where constant $B$ (which is related to Hamaker constant ${ }^{25}$ ) can be fitted to DFT data, or can be simply estimated from the equation ${ }^{26}$

$$
B=\left(\rho_{2 \infty}-\rho_{1 \infty}\right)\left(\rho_{3} u_{3}-\rho_{2 \infty} t_{3}\right),
$$

where $\rho_{1 \infty}$ and $\rho_{2 \infty}$ are vapor and liquid densities at phase coexistence and coefficients $u_{3}$ and $t_{3}$ are integration characteristics of intermolecular potentials. Here $u_{3}$ stems from attractive contribution of the substrate potential, while $t_{3}$ is due to van der Waals interactions between liquid molecules. This means that the term $\rho_{3} u_{3}$ exists only in the case of heteroge- neous nucleation and depends on the nature of interaction between the solid substrate and liquid, while term $-\rho_{2 \infty} t_{3}$ contributes the disjoining pressure even in the case of homogeneous nucleation. Both $u_{3}$ and $t_{3}$ are positive, but $\rho_{3} u_{3}$ is much greater than the term $\rho_{2 \infty} t_{3}$, yielding a positive constant $B$ and a positive disjoining pressure. Positive and monotonic disjoining pressure versus the film thickness has profound effect on heterogeneous nucleation. It gives a nonmonotonic behavior for chemical potential of liquid condensate versus liquid film thickness and is responsible for the existence of stable equilibrium liquid clusters on the surface of wettable particles.

Without the solid particles the $u_{3}$ term is zero and the main contribution to $B$ [Eq. (13)] is a negative van der Waals term. Hence, $B$ is negative and so is the disjoining pressure. In the homogeneous nucleation the thickness of the fluid film is the same as the radius of the droplet, as $R_{n}=0$ and $R$ $=R_{n}+h=h$. As such, one cannot use the planar limit to estimate the disjoining pressure since it strongly depends on the droplet radius. In addition to van der Waals contribution, the disjoining pressure may depend on other properties, especially when the thickness of the liquid film or the size of the droplet is on the same order of molecular size. ${ }^{1}$

Let us consider an incompressible liquid that can form a thin film of thickness $h$ on the surface of the particle of radius $R_{n}$ (here we ignore the difference between $R_{n}$ and $\widetilde{R}_{n}$ for the purpose of simple estimation). The $R_{n}$ value can range from microscopic to mesoscopic. If one neglects all the interaction between the liquid and solid particles and only accounts for the van der Waals attraction in liquid, the intermolecular potential can be presented as

$$
w(r)=-\frac{\lambda}{r^{6}},
$$

where $\lambda$ is a positive constant.

To analyze the effect of the disjoining pressure, we replace the bulk liquid of density $\rho_{2}$ around the solid particle of radius $R_{n}$ by a thin liquid film of the same density and a vapor of density $\rho_{1}$ for $r>R$. Thus, the disjoining pressure is equal to the difference in interaction energy per unit volume between these two configurations

$$
\Pi=-\rho_{2} \int_{\text {sur }} d V\left(\rho_{2}-\rho_{1}\right) \frac{\lambda}{r^{6}},
$$

where the volume integration is taken over the space surrounding the droplet. To calculate this integral we used the cylindrical coordinate system depicted in Fig. 1. Here the distance between two molecules is $r=\sqrt{(z-R+h)^{2}+y^{2}}$, so the integral becomes

$$
\begin{aligned}
\Pi= & -\rho_{2}\left(\rho_{2}-\rho_{1}\right) \\
& \times 2 \pi \lambda\left(\int_{-\infty}^{-R} d z \int_{0}^{\infty} y d y \frac{1}{\left((z-R+h)^{2}+y^{2}\right)^{3}}\right. \\
& +\int_{-R}^{+R} d z \int_{\sqrt{R^{2}-z^{2}}}^{\infty} y d y \frac{1}{\left((z-R+h)^{2}+y^{2}\right)^{3}}
\end{aligned}
$$




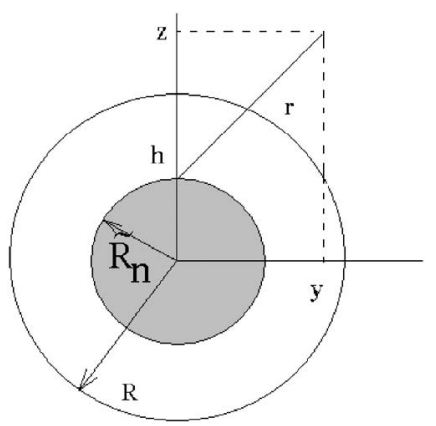

FIG. 1. A schematic plot of a thin liquid film formed on a spherical solid particle.

$$
\left.+\int_{+R}^{+\infty} d z \int_{0}^{\infty} y d y \frac{1}{\left((z-R+h)^{2}+y^{2}\right)^{3}}\right)
$$

After this integration the disjoining pressure is given by

$$
\Pi=-\rho_{2}\left(\rho_{2}-\rho_{1}\right) \lambda \frac{4 \pi}{3} \frac{R^{3}}{(2 R-h)^{3} h^{3}} .
$$

Note that $\Pi$ is a function of two variables $R$ and $h$. So we consider two special cases. The first one is for heterogeneous nucleation on impurity particles of a very large size, which was considered in Ref. 1 . In this case we only need to consider $R_{n} \gg h$, because if $h$ is comparable to $R_{n}, \Pi$ is negligible. Hence, Eq. (17) can be expanded in terms of small parameter $h / R_{n}$ (or $h / R$ ). The zero-order contribution gives

$$
\begin{aligned}
\Pi & =-\rho_{2}\left(\rho_{2}-\rho_{1}\right) \lambda \frac{4 \pi}{3} \frac{1}{(2-h / R)^{3} h^{3}} \\
& \approx-\rho_{2}\left(\rho_{2}-\rho_{1}\right) \lambda \frac{4 \pi}{3} \frac{1}{8 h^{3}} .
\end{aligned}
$$

This estimation is equivalent to that given by the $-\rho_{2 \infty} t_{3}$ term in Eq. (13), that is, to replace the disjoining pressure by its value in the planar limit. ${ }^{1}$ This replacement is quite accurate, since the van der Waals term $-\rho_{2 \infty} t_{3}$ in Eq. (13) is much smaller than the first contribution from the $\rho_{3} u_{3}$ term.

The second special case is for homogeneous nucleation without the presence of impurity particles, so that $R_{n}=0$ and $R=h$. Thus, Eq. (17) becomes

$$
\Pi=-\rho_{2}\left(\rho_{2}-\rho_{1}\right) \lambda \frac{4 \pi}{3} \frac{1}{R^{3}} .
$$

If one ignores vapor density $\rho_{1}$ as $\rho_{1} \ll \rho_{2}$, it gives the expression for disjoining pressure of the small droplet, obtained earlier by Tsekov et al. ${ }^{17}$ Note that the disjoining pressure in this case is eight times larger compared to that of the planar thin film [Eq. (18)], assuming $R=h$.

In the case of microscopic wettable particles, for which both $R$ and $h$ are comparable, the disjoining pressure can only be estimated based on Eq. (17), not mentioning other complicated contributions which are not accounted for in this simple estimation of the disjoining pressure. In the following sections we apply DFT to obtain more quantitative values of the disjoining pressure.

\section{DFT CALCULATION OF MODEL SYSTEM}

Again, we used the DFT/WDA approach to study the inhomogeneous system of microscopic wettable particle surrounded by a fluid film. The homogeneous nucleation is also considered as a special case of heterogeneous nucleation with $R_{n}=0$, even though the DFT treatment of homogeneous nucleation does not require WDA (since LDA is sufficient). We will compare results based on both LDA and WDA to see whether WDA can bring some new insights into homogeneous nucleation problem. For example, it is known that small oscillations of liquid density may occur inside of the planar liquid-vapor interface even without the solid substrate. $^{27}$ In Ref. 1 we discussed how this densityoscillation behavior can affect the planar surface tension and the Tolman length. Here we will see whether similar effect can change the surface tension of small liquid droplet.

First, we briefly summarize main aspects of DFT/LDA and DFT/WDA (Refs. 18 and 19) theoretical formalisms. Let $w\left(\mathbf{r}_{1}, \mathbf{r}_{2}\right)=w\left(r_{12}\right)$ represent the pairwise intermolecular potential for the simple nonpolar molecules. In DFT the free energy of a nonuniform system, within random-phase approximation (RPA), is given by

$$
F[\rho]=\int d \mathbf{r} f(\rho(r))+\frac{1}{2} \iint d \mathbf{r}_{1} d \mathbf{r}_{2} w_{p}\left(r_{12}\right) \rho\left(r_{1}\right) \rho\left(r_{2}\right),
$$

where $\rho(\mathbf{r})=\rho(r)$ is the density profile for spherically symmetrical system (the $r$ value is measured from the center of the droplet) and $w_{p}\left(r_{12}\right)$ is the weak attractive part of intermolecular potential. We then approximate the repulsive part of free energy density $f(\rho)$ either with the free energy density of hard spheres $f_{\mathrm{hs}}(\rho(r))$ in Carnahan-Starling form ${ }^{28}$ in LDA or with $f(\rho(r))=f_{\text {id }}(\rho(r))+\rho(r) \Delta \psi_{\text {hs }}(\bar{\rho})$ in WDA. Here $f_{\text {id }}(\rho(r))$ is the free energy density of ideal gas and $\Delta \psi_{\mathrm{hs}}(\bar{\rho})$ is the excess free energy density in Carnahan-Starling form. ${ }^{28}$ This $\Delta \psi_{\mathrm{hs}}(\bar{\rho})$ is a function of weighted density $\bar{\rho}(r)$. The latter can be determined by averaging the true local density $\rho(r)$ over certain local volume. Following Tarazona ${ }^{18}$ and Tarazona et al., 19

$$
\bar{\rho}\left(r_{1}\right)=\int d \mathbf{r}_{2} \rho\left(r_{2}\right) \omega\left(r_{12}, \bar{\rho}\left(r_{1}\right)\right),
$$

where the weighting function $\omega$ is chosen according to the model of Tarazona ${ }^{18}$ and Tarazona et al.. ${ }^{19}$

The open system is considered in grand canonical ensemble. In DFT the grand thermodynamic potential is given by

$$
\Omega[\rho]=F[\rho]+\int d \mathbf{r}_{1} \rho\left(r_{1}\right) V_{\mathrm{ext}}\left(r_{1}\right)-\mu \int d \mathbf{r}_{1} \rho\left(r_{1}\right),
$$

where $V_{\text {ext }}\left(r_{1}\right)$ is the external potential. In the case of heterogeneous nucleation this external potential arises due to the impurity particle at the center of the droplet. For homogeneous nucleation this potential is zero. The equation for equilibrium density profile can be derived based on the variation condition $\delta \Omega[\rho] / \delta \rho=0$. If one uses LDA (for homogeneous nucleation with $V_{\text {ext }}=0$ ), the variation gives 


$$
\mu_{h}\left(\rho\left(r_{1}\right)\right)=\mu-\frac{2 \pi}{r_{1}} \int_{0}^{\infty} r_{2} d r_{2} \rho\left(r_{2}\right) \int_{\left|r_{1}-r_{2}\right|}^{\left|r_{1}+r_{2}\right|} r_{12} d r_{12} w_{p}\left(r_{12}\right)
$$

Using WDA one can obtain

$$
\begin{aligned}
\rho\left(r_{1}\right)= & \exp \left\{-\frac{1}{k_{B} T}\left[\frac{2 \pi}{r_{1}} \int_{0}^{\infty} r_{2} d r_{2} \rho\left(r_{2}\right) \Delta \psi_{\mathrm{hs}}^{\prime}\left(\bar{\rho}\left(r_{2}\right)\right)\right.\right. \\
& \times \int_{\left|r_{1}-r_{2}\right|}^{\left|r_{1}+r_{2}\right|} r_{12} d r_{12} \frac{\omega\left(r_{12}, \bar{\rho}\left(r_{2}\right)\right)}{1-\bar{\rho}_{1}\left(r_{2}\right)-2 \bar{\rho}_{2}\left(r_{2}\right) \bar{\rho}\left(r_{2}\right)} \\
& +\Delta \psi_{\mathrm{hs}}\left(\bar{\rho}\left(r_{1}\right)\right)+\frac{2 \pi}{r_{1}} \int_{0}^{\infty} r_{2} d r_{2} \rho\left(r_{2}\right) \\
& \left.\left.\times \int_{\left|r_{1}-r_{2}\right|}^{\left|r_{1}+r_{2}\right|} r_{12} d r_{12} w_{p}\left(r_{12}\right)+V_{\mathrm{ext}}\left(r_{1}\right)-\mu\right]\right\},
\end{aligned}
$$

where $\bar{\rho}_{1}$ and $\bar{\rho}_{2}$ are defined by Tarazona ${ }^{18}$ and Tarazona et al. ${ }^{19}$

To solve the last two equations numerically, the exact potential function should be known. Again, we used the Lennard-Jones (LJ) model potential, for which the attractive part of intermolecular potential $w_{p}\left(r_{12}\right)$ is approximated by using Weeks-Chandler-Anderson perturbation scheme [see Eq. 19 in Ref. 1].

For homogeneous nucleation, it can be shown that Eq. (23) has three solutions: two uniform solutions $\rho_{1}$ and $\rho_{2}$ representing the bulk vapor and liquid, and a nonuniform solution $\rho(r)$ representing the spherical density profile of the (unstable) critical droplet. The latter refers to the saddle point of the grand thermodynamic potential. We followed the same numerical iterational procedure ${ }^{23}$ to solve Eq. (23) as well as Eq. (24). For Eq. (24), the numerical solution is more challenging to derive. The procedure has to be adjusted, using the technique introduced by Tarazona. ${ }^{18}$ One has to choose an appropriate initial density profile and to conduct iterations very carefully to monitor changes of $\Omega$. This numerical procedure becomes even harder to carry out for low supersaturations, at which the size of the critical cluster is large but not very sensitive to the supersaturation.

It is worthy to note that at low temperature $T^{*}$ $=k_{B} T / \epsilon_{\mathrm{LJ}}=0.7\left(\epsilon_{\mathrm{LJ}}\right.$ is a parameter of LJ potential $)$, the density profiles obtained from Eq. (24) still exhibit weak oscillations on the liquid side of the interface as in the planar case, even though the surface layer is much thinner now.

For heterogeneous nucleation with impurity particles of any size, a particle-liquid interaction potential function $V_{\text {ext }}(r)$ is required. Here, we still used the same potential as in Ref. 1, that is,

$$
V_{\mathrm{ext}}(r)=-\rho_{3}\left(\frac{u_{3}}{\left(r-\widetilde{R}_{n}\right)^{3}}+\frac{u_{9}}{\left(r-\widetilde{R}_{n}\right)^{9}}\right),
$$

with $\rho_{3}=12 / d^{3}, u_{3}=2.348 \epsilon_{\mathrm{LJ}} \sigma^{6}$, and $u_{9}=-5.326 \epsilon_{\mathrm{LJ}} \sigma^{12}$ as in Ref. 26. For numerical calculation we set $V_{\text {ext }}(r)=\infty$ for $r$ $<\widetilde{R}_{n}+0.84 d$. Calculations were performed for the given $T^{*}$ $=0.7$. Although Eq. (24) also has three solutions, all of them are nonuniform due to the presence of the impurity particle. The solutions are as follows. (1) Equilibrium cluster with thin liquid film on the surface of the particle. This is a stable solution resulting from the minimization of the thermodynamic potential. (2) Critical cluster of the liquid droplet with solid particle at the center, which is in unstable equilibrium with the surrounding vapor. (3) Infinitely thick bulklike liquid surrounding the solid particle. To start with the numerical iteration procedure, the initial step function should mimic two possible dropletlike solutions as close as possible. We calculated density profiles for several given chemical potentials. The density profiles are harder to obtain as the chemical potential approaches to its threshold value, where the difference between two solutions is nearly undistinguishable. Whenever the threshold supersaturation is reached, heterogeneous nucleation becomes barrierless. Nevertheless, it is very difficult to find accurate threshold value of chemical potential, since the solution is highly unstable when dealt with the iteration procedure.

As expected, the density profiles exhibit a strong oscillatory behavior near the surface of the solid particle. Since we are mainly interested in microscopic particles here, we performed calculations for two sizes: (1) $\widetilde{R}_{n}=5.0 d$ which is somewhat close to mesoscopic size, and (2) $\widetilde{R}_{n}=3.5 d$ which is surely a microscopic size, but still much larger than the molecular size.

We considered a number of values of chemical potentials (supersaturations) within the range $\mu_{\max }>\mu>\mu_{\infty}$. The maximum value of the chemical potential $\mu_{\max }=\mu_{s 1}$ is the spinodal value, above which the only equilibrium solution is the uniform liquid in the case of homogeneous nucleation. The maximum value of the chemical potential in the case of heterogeneous nucleation is the threshold value $\mu_{\max }=\mu_{\mathrm{th}}$. Nucleation becomes barrierless when $\mu>\mu_{\mathrm{th}}$. The minimum value of the chemical potential $\mu=\mu_{\infty}$ corresponds to vaporliquid coexistence, where vapor and liquid bulk pressures are equal $p_{1}=p_{2}=p_{\infty}$. For $\mu>\mu_{\infty}$ we have liquid pressure $p_{2}$ $>p_{1}$ which leads to metastability of vapor phase. Based on the density profile solutions obtained by solving Eqs. (23) and (24) with the chemical potential in the range $\mu_{\max }>\mu$ $>\mu_{\infty}$, we can study properties of the liquid droplets and kinetics of liquid-vapor nucleation. These properties are discussed in the following sections.

\section{HOMOGENEOUS NUCLEATION}

We proceeded with a definition of the droplet's radius $R$ (or the dividing surface). In fact, thermodynamic quantities on the right-hand side of Eq. (6) are strongly dependent on the choice of the dividing surface, and yet the definition of the dividing surface is not unique for droplets. The most common definition is such that the dividing surface can be solely determined by the density profile. For instance, we can use the equimolar dividing surface, which is defined such that the adsorption $\Gamma_{21}=0$. Then, according to the Gibbs method of the dividing surfaces, the number of particles in a droplet can be given by 


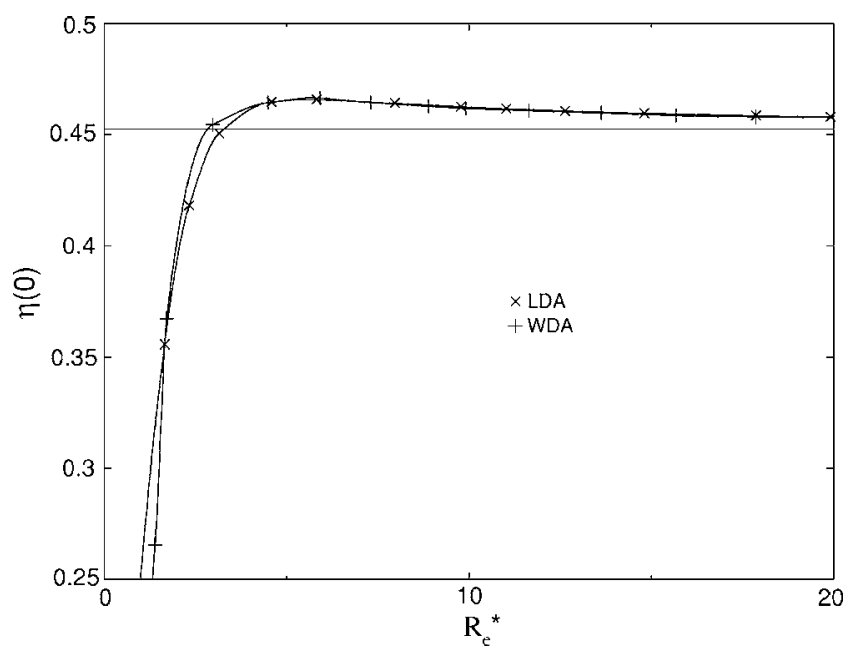

FIG. 2. The dependence of the reduced density $\eta(0)=\pi d^{3} \rho(0) / 6$ at the center of a homogeneous droplet on the dimensionless equimolar radius $R_{e}^{*}=R_{e} / d$ of the droplet at $T^{*}=k_{B} T / \epsilon_{\mathrm{LJ}}=0.7$. The horizontal line represents the liquid density at phase coexistence.

$$
4 \pi \int_{0}^{L} r^{2} \rho(r) d r=\frac{4 \pi}{3} R_{e}^{3} \rho_{2}+\frac{4 \pi}{3}\left(L^{3}-R_{e}^{3}\right) \rho_{1},
$$

where $R=R_{e}$ is the radius of equimolar surface. This equation can be rewritten as

$$
R_{e}^{3}=\frac{3 \int_{0}^{L} r^{2}\left(\rho(r)-\rho_{1}\right) d r}{\rho_{2}-\rho_{1}} .
$$

Clearly, the equimolar surface is only dependent on the density profile and bulk $\rho_{1}$ and $\rho_{2}$ values, thus it is unique.

With the dividing surface $R$ defined we turn our attention to the main objective of this section-properties of droplets as a function of droplet size. It is known that the higher the supersaturation (or $\mu$ ), the smaller the size (or radius $R$ ) of the critical droplet, and the higher density of bulk liquid due to compressibility factor. The density at the center of the critical droplet, however, only behaves this way at low supersaturations (see Fig. 2). In contrast, for extremely small droplets, the center density decreases considerably at certain size. It can only be explained by overlapping of the surface layer of the droplet. This behavior can be seen from both LDA and WDA calculations of the density profile (Fig. 2), as well as from the gradient-expansion calculation. ${ }^{29}$ Note that the weighted density profile is used to illustrate density at the center of the droplet in WDA calculation (to exclude secondary effect of density oscillation about the weighted value.) At the spinodal, the critical cluster completely turns into uniform vapor $\left(\rho(r)=\rho_{1 s}\right)$ and $R_{e}=0$ according to Eq. (27).

\section{A. Disjoining pressure $\Pi(R)$}

For a given $\mu$ in between $\mu_{\infty}$ and $\mu_{s 1}$, the disjoining pressure $\Pi(R)$ can be calculated on the basis of the definition Eq. (9) in which the bulk liquid pressure $p_{2}$ is known. The normal component of the pressure tensor $p_{N}(0)$ at the center of the droplet can be calculated based on the general mechanical relation $p_{N}(0)=p_{T}(0)$ in the case of spherical symmetry (e.g., Ref. 16). Meanwhile, the tangential component of the pressure tensor is equal to the negative density of the
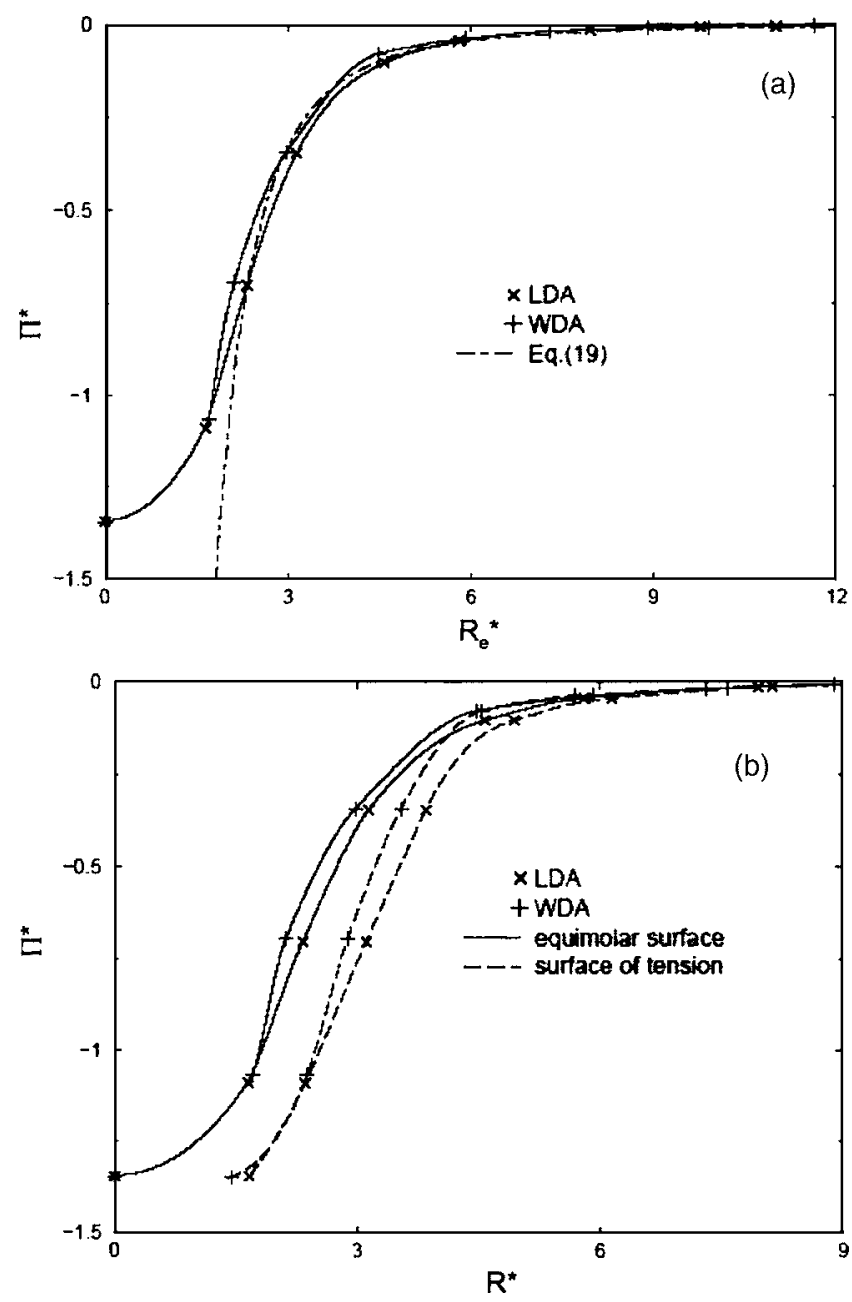

FIG. 3. (a) The reduced disjoining pressure $\left(\Pi^{*}=\Pi \pi d^{3} / 6 k_{B} T\right)$ of small homogeneous droplets vs the equimolar dividing surface $\left(R_{e}^{*}=R_{e} / d\right)$ at $T^{*}$ $=k_{B} T / \epsilon_{\mathrm{LJ}}=0.7$. (b) The reduced disjoining pressure $\left(\Pi^{*}=\Pi \pi d^{3} / 6 k_{B} T\right)$ of small homogeneous droplets vs two different dividing surfaces $\left(R_{E}^{*}=R_{e} / d\right.$ and $\left.R^{*}=R / d\right)$ at $T^{*}=k_{B} T / \epsilon_{\mathrm{LJ}}=0.7$.

grand thermodynamic potential which is readily calculated from DFT. Eventually, one can obtain the relation between the disjoining pressure and equimolar radius of the critical droplet. This relation is presented in Fig. 3(a), obtained from both LDA and WDA calculations. Both calculations show that in the case of small droplets the disjoining pressure is negative and increases in absolute value with decreasing the radius of the droplet. In the same figure, we also plotted the estimated $\Pi(R)$ [Eq. (19)], where $\lambda$ was taken to be $4 \epsilon_{\mathrm{LJ}} \sigma_{\mathrm{LJ}}$ for the Lennard-Jones potential. The simple estimation agrees reasonably well with the DFT numerical results, at least for $R>3 d$, even though neither the compressibility of liquid nor overlapping of the surface layer was taken into account. For the smaller droplets (less than $3 d$ in radius) the overlapping of the surface layer is much stronger and it is mainly responsible for the difference between the computed $\Pi$ and the estimated one from Eq. (19). Similar behavior was seen in Ref. 1, where we showed that power-law approximation works well for thick liquid films, but an exponential approximation has to be used for thinner structural films due to strong overlapping of the surface layer. 


\section{B. Surface tension}

There are two ways to define the surface tension of small homogeneous droplets, depending on whether the disjoining pressure contribution is considered as part of the surface tension term or not. To calculate the surface tension from DFT, we rewrite Eq. (6) as

$$
\sigma_{21}=\frac{\Omega+p_{1} 4 \pi L^{3} / 3}{4 \pi R^{2}}+\left(p_{2}-p_{1}\right) \frac{R}{3}-\int_{R}^{L} \frac{r^{2}}{R^{2}} \Pi(r) d r,
$$

where the grand thermodynamic potential $\Omega$ can be calculated according to Eq. (22), and the bulk pressures $p_{1}$ and $p_{2}$ are known for a given value of $\mu$, as well as the disjoining pressure $\Pi(r)$.

An alternative way to define the surface tension is to include the disjoining pressure term. As such, one obtains the traditional thermodynamic surface tension

$$
\sigma_{21 t}=\frac{\Omega+p_{1} 4 \pi L^{3} / 3}{4 \pi R^{2}}+\left(p_{2}-p_{1}\right) \frac{R}{3} .
$$

In principle, both definitions entail arbitrary radius of dividing surface $R$. If the radius is chosen such that it is independent of the definition of surface tension, then there is a simple relationship between both surface tensions

$$
\sigma_{21}=\sigma_{21 t}-\int_{R}^{L} \frac{r^{2}}{R^{2}} \Pi(r) d r .
$$

In the previous section, we have shown that the disjoining pressure is negative for small droplet. This means that the surface tension $\sigma_{21}$ calculated according to Eq. (28) is always higher than $\sigma_{21}$.

Let us first consider a few limiting cases. When droplets become very large, the disjoining pressure decreases rapidly and the last term in Eqs. (28) and (30) diminishes. Thus, both surface tensions approach to the same limiting value of planar liquid-vapor surface tension which is independent of the dividing surface.

When the chemical potential approaches to the value of liquid spinodal, the equimolar size of the droplet approaches to zero. In such a case the first term in Eq. (29) vanishes, since $\Omega$ is equal to its bulk value $-p_{1}(4 \pi / 3) L^{3}$. The second term also becomes zero as $R=0$ and thus, thermodynamic surface tension calculated according Eq. (29) is zero. On the other hand, the surface tension defined via Eq. (28) diverges as $1 / R^{2}$. Note that this effect occurs for equimolar dividing surface since the droplet completely disappears in vapor at the spinodal, that is, the liquid-vapor interface is effectively in nonexistence, while the surface energy per unit of area of the droplet grows infinitely in order to compensate the effect of the disjoining pressure.

Figure 4(a) shows the dependence of the surface tension on the equimolar radius of the droplet based on both WDA and LDA calculations. Thermodynamic surface tension calculated according to Eq. (29) is a nonmonotonic function of the droplet size. It becomes slightly higher than the planar value as the size of the droplet decreases, exhibits a maximum at intermediate radius around 7-9d, and eventually approaches to zero as the size of the droplet goes to zero.
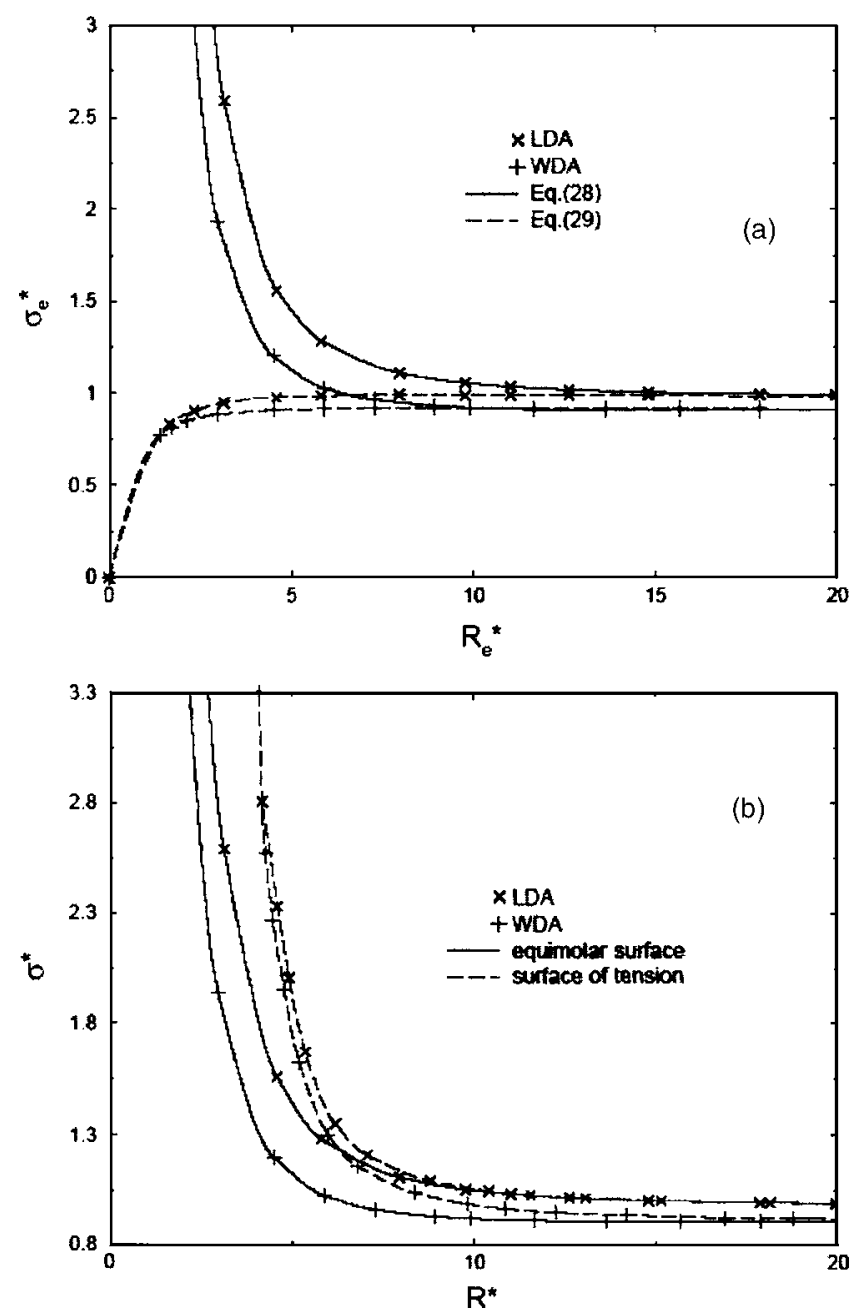

FIG. 4. (a) The reduced surface tension $\left(\sigma_{e}^{*}=\sigma_{21 e} \pi d^{2} / 6 k_{B} T\right)$ of small homogeneous droplets vs the equimolar dividing surface $\left(R_{e}^{*}=R_{e} / d\right)$ at $T^{*}$ $=k_{B} T / \epsilon_{\mathrm{LJ}}=0.7$. (b) The reduced surface tension $\left(\sigma^{*}=\sigma_{21} \pi d^{2} / 6 k_{B} T\right)$ of small homogeneous droplets vs two different dividing surfaces at $T^{*}=k_{B} T / \epsilon_{\mathrm{LJ}}$ $=0.7$.

Both LDA and WDA give similar behavior, although the more accurate WDA gives smaller surface tension and weaker nonmonotonic behavior than LDA. In contrast, the surface tension defined based on Eq. (28), which excludes the disjoining pressure effect, shows a monotonic increase from large to small droplets due to the negative value of the Tolman length in the planar limit.

The existence of multiple definitions of radius of the droplet has caused debates in the theory of curved surfaces, especially on the dependence of surface tension to the curvature. There is another common definition of radius, namely, the radius of surface of tension, which is not unique. The surface of tension is defined such that the Laplace equation (8) can be expressed in a simple form

$$
p_{2}-p_{1}=\frac{2 \sigma_{21 s}}{R_{s}},
$$

where $\sigma_{21 s}$ refers to the surface tension at the surface of tension and $R_{s}$ refers to the radius of the surface of tension. Thus, one has 


$$
\frac{\partial \sigma_{21 s}}{\partial R}=\Pi\left(R_{s}\right)
$$

and

$$
\sigma_{21 s}=\frac{\left(p_{2}-p_{1}\right) R_{s}}{2} .
$$

Substituting Eq. (33) into Eq. (28) gives

$$
R_{s}^{3}=\frac{6\left(\Omega-4 \pi / 3 L^{3} p_{1}\right)}{4 \pi\left(p_{2}-p_{1}\right)}-6 \int_{R_{s}}^{L} r^{2} \frac{\Pi(r)}{p_{2}-p_{1}} d r .
$$

The solution of Eq. (34) is the radius of surface of tension. The equation can be solved numerically using iteration method. For very large droplets the second term on the right hand is almost negligible and the first term can provide an accurate estimation for the radius of tension (which is also the classical thermodynamic definition of this radius). This estimation of $R_{S}$ can then be inserted into the second term and a new approximation for the radius can be obtained. After several iterations the radius can be calculated with high accuracy. For large droplets we have $R_{s}>R_{e}$ because the Tolman length is a negative quantity in the limit of the large droplets. For smaller droplets, the first term on the right-hand side of Eq. (34) becomes smaller and vanishes in the smalldroplet limit while the second term remains finite. This means that the radius of tension calculated according to Eq. (34) is nonzero even at the liquid spinodal and that the surface tension according to Eq. (33) still remains finite. Figure 3(b) shows how the disjoining pressure depends on the radius of surface of tension $R_{s}$. $\Pi\left(R_{s}\right)$ is still a monotonic function but the curve is shifted to the right in the region of the small droplet sizes. Figure 4(b) shows the surface tension calculated according to Eq. (33) as a function of $R_{s}$. Both WDA and LDA curves are monotonic and close to each other. The curves are shifted to the right compared to those obtained based on the equimolar dividing surface.

As mentioned above, definition of the surface of tension is not unique. Another definition can be based on the Laplace equation in the form of Eqs. (10) and (11). Equation (11) provides the first term on the right-hand side of Eq. (34), which only agrees with the full version of this equation at low supersaturations (large droplets). If one uses Eq. (10) as the Laplace equation and sets

$$
p_{N}(0)-p_{1}=\frac{2 \sigma_{21 s}}{R_{s}},
$$

then Eq. (34) becomes

$$
R_{s}^{3}=\frac{1}{p_{2}-p_{1}+3 \Pi} \frac{6\left(\Omega-4 \pi / 3 L^{3} p_{1}\right)}{4 \pi}-6 \int_{R_{s}}^{L} r^{2} \Pi(r) d r .
$$

It can be seen that Eq. (36) yields negative value for $R_{s}$ at high supersaturations, due to overlapping of the surface layer, when $p_{2}-p_{1}$ becomes less than $3|\Pi|$. It seems that Eq. (36) may not be the right way to introduce the surface of tension. However, Eq. (35) is often used to calculate mechanical surface tension and to define the radius of mechani- cal surface of tension. This may be one of the reasons why mechanical and thermodynamic definitions of the surface tension and the Tolman length give different results for the small droplets. For large droplets, when disjoining pressure is negligible, all definitions of the surface of tension give the same result.

In summary, the radius of equimolar dividing surface appears to be more reliable to describe the size of the droplet, since it is independent of the surface tension and the disjoining pressure. We use this radius hereafter.

\section{Chemical potential of the liquid condensate}

A prerequisite to calculate the barrier height to nucleation is the chemical potential of the liquid condensate as a function of the critical droplet radius $R$ or the number of particles $\nu$ in the critical droplet. In fact, the definition of the surface tension and the disjoining pressure should not affect the experimentally measurable thermodynamic characteristics such as chemical potential. We showed in Ref. 1 that the presence of disjoining pressure in heterogeneous droplets results in a nonmonotonic behavior of $\mu(\nu)$ (see Fig. 4 in Ref. 1). The disjoining pressure also occurs in homogeneous droplets. We will examine whether it will cause a nonmonotonic behavior of the chemical potential or not. Note that the classical nucleation theory does not predict such nonmonotonic behavior. However, the fact that the nucleation rates calculated based on the capillary approximation differ from the measured nucleation rates at high supersaturations led some researchers ${ }^{30,31}$ to speculate that chemical potential of the liquid condensate may be a nonmonotonic function of $\nu$ even for homogeneous nucleation. In Fig. 5(a), we plot chemical potential as a function of particle number $\nu$ (within the equimolar dividing surface) using both LDA and WDA. To calculate $\nu$, we used the equation

$$
\nu=\frac{4 \pi}{3} R^{3} \rho_{2}+4 \pi R^{2} \Gamma_{21}=\frac{4 \pi}{3} R_{e}^{3} \rho_{2},
$$

where the adsorption $\Gamma_{21}=0$ at the equimolar dividing surface. We also plot the curve based on the capillary approximation

$$
\Delta \mu=\frac{2 \sigma_{21 \infty}}{\rho_{2 \infty} R} .
$$

One can see that all the $\mu(\nu)$ curves are monotonic. Hence we conclude that the monotonic behavior of chemical potential of the critical droplets is a distinct feature of homogeneous nucleation. This feature characterizes a qualitative difference between the homogeneous and heterogeneous nucleations. On the other hand, from Fig. 5(a) one can see a large difference among predictions of capillary approximation [Eq. (38)] and DFT for small droplets. In fact, the higher the supersaturation (the chemical potential of vapor), the larger differences between predictions of classical capillary approximation and DFT calculation. The DFT curve is much lower than that based on Eq. (38). At the spinodal the classical theory fails to predict the existence of threshold chemical potential at which the barrier becomes zero. For large droplets, the capillary-approximation curve and the LDA 

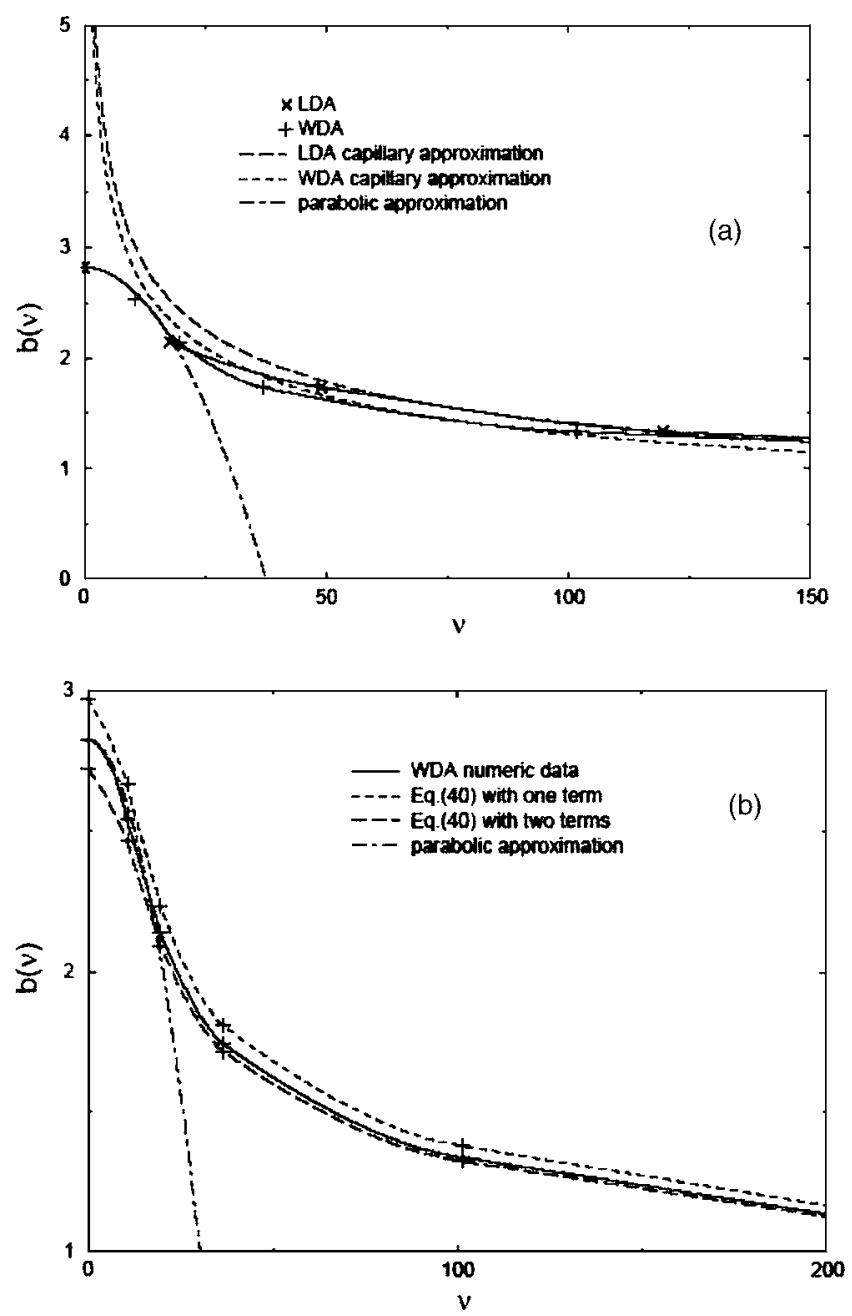

FIG. 5. (a) The dependence of the reduced chemical potential of the liquid condensate $b=\left(\mu-\mu_{\infty}\right) / k_{B} T$ on the number of particles $\nu$ of small homogeneous droplets at $T^{*}=k_{B} T / \epsilon_{\mathrm{LJ}}=0.7$. (b) The dependence of the reduced chemical potential of the liquid condensate $b=\left(\mu-\mu_{\infty}\right) / k_{B} T$ on the number of particles $\nu$ of small homogeneous droplets at $T^{*}=k_{B} T / \epsilon_{\mathrm{LJ}}=0.7$.

curve are located higher than the WDA curve. This is because WDA predicts lower planar surface tension than LDA (at least far below critical temperature).

More insights into the behavior of chemical potential can be gained from the relation between the chemical potential and the pressure in liquid condensate. The pressure difference $p_{2}-p_{1}$ can be calculated via the compressibility route, as shown before in Eq. (37) of Ref. 1, that is,

$$
p_{2}-p_{1}=p_{1 \infty}-p_{1}+\rho_{2 \infty} \Delta \mu+\frac{1}{2} \rho_{2 \infty}^{2} \chi_{2} \Delta \mu^{2},
$$

where $\chi_{2}$ is the compressibility of liquid. In Eq. (39) the term $p_{1 \infty}-p_{1}$ can be neglected compared to the next two terms as $\rho_{1} \ll \rho_{2}$ (below the critical temperature). By neglecting $p_{1 \infty}$ $-p_{1}$ and expanding over small parameter $\chi_{2}\left(p_{2}-p_{1}\right)$, we obtained

$$
\Delta \mu=\frac{\left(p_{2}-p_{1}\right)}{\rho_{2 \infty}}-\frac{1}{2} \chi_{2} \frac{\left(p_{2}-p_{1}\right)^{2}}{\rho_{2 \infty}} .
$$

In Eq. (40) the first term is the main term and the second term is the correction term due to liquid compressibility. This equation shows that the chemical potential is largely propor- tional to the pressure difference between liquid and vapor phases. The pressure difference can be determined from the Laplace equation (8). In the case of heterogeneous nucleation $^{1}$ the disjoining pressure term in this equation is negative, causing chemical potential to be a nonmonotonic function of the droplet size. In the case of homogeneous nucleation this term is positive. In contrast to mesoscopic droplets, for which the surface tension is only slightly affected by the small Tolman correction ${ }^{1}$, now the surface tension is strongly dependent on the curvature. Thus, this strong curvature dependence of the surface tension in the first two terms of the Laplace equation (8) gives an explanation to the difference in the calculated chemical potential from the capillary approximation and DFT [Fig. 5(b)]. The approximation of Eq. (40) slightly overestimates the threshold value if one term is considered, but underestimates it if two terms are considered. By and large, Eq. (40) is in good agreement with numerical results. Note that the values of bulk pressures $p_{1}$ and $p_{2}$, compressibility, and liquid density are all measurable quantities, and thus experimental data can be used in Eq. (40).

When the chemical potential of the liquid condensate is close to its maximum threshold value, one can introduce the near-threshold region defined by condition of Eq. (57) in Ref. 1. In this region the nucleation rate is sufficiently different from zero and the $\mu(\nu)$ curve for the critical clusters can be approximated by parabolic one, that is,

$$
b(\nu) \approx b_{s 1}-\frac{1}{2} A \nu^{2},
$$

where $b(\nu)=\Delta \mu(\nu) / k_{B} T$ is the reduced chemical potential which is dependent on two parameters: (1) $b_{\max }=b_{s 1}$, the spinodal value of chemical potential which can be calculated from the bulk properties, and (2) $A=\left|d^{2} b(\nu) / d \nu^{2}\right|_{0}$, which can be fitted to DFT curves in Fig. 5. The curves fitting from Eq. (41) are also shown in Fig. 5. At the reduced temperature $T^{*}=k_{B} T / \epsilon_{\mathrm{LJ}}=0.7$, the fitting value $A=0.0040$. This parabolic approximation works well for very small clusters with less than 25 particles. We will use it to calculate the free energy of droplet formation in the near-threshold region.

\section{Free energy of droplet formation and nucleation barrier height}

The free energy of droplet formation and the barrier height are key characteristics that determine the rate of nucleation. $^{1,32,33}$ Following the same approach as in Ref. 1 we computed the free energy of droplet formation for droplet of any size on the basis of $b(\nu)$ for critical clusters (derived in the previous subsection). When a liquid droplet is in mechanical and thermal equilibriums with the surrounding vapor, one has

$$
\partial W / \partial \nu=b(\nu)-b_{1},
$$

where $W$ is the reduced free energy of droplet formation (in units of $k_{B} T$ ) and $b_{1}$ is the reduced chemical potential of vapor surrounding liquid cluster. To derive the formation free energy as a function of the size of the droplet, we integrated Eq. (42) with the boundary condition $W(0)=0$ (the formation free energy is zero if the size of droplet is zero) and obtained 

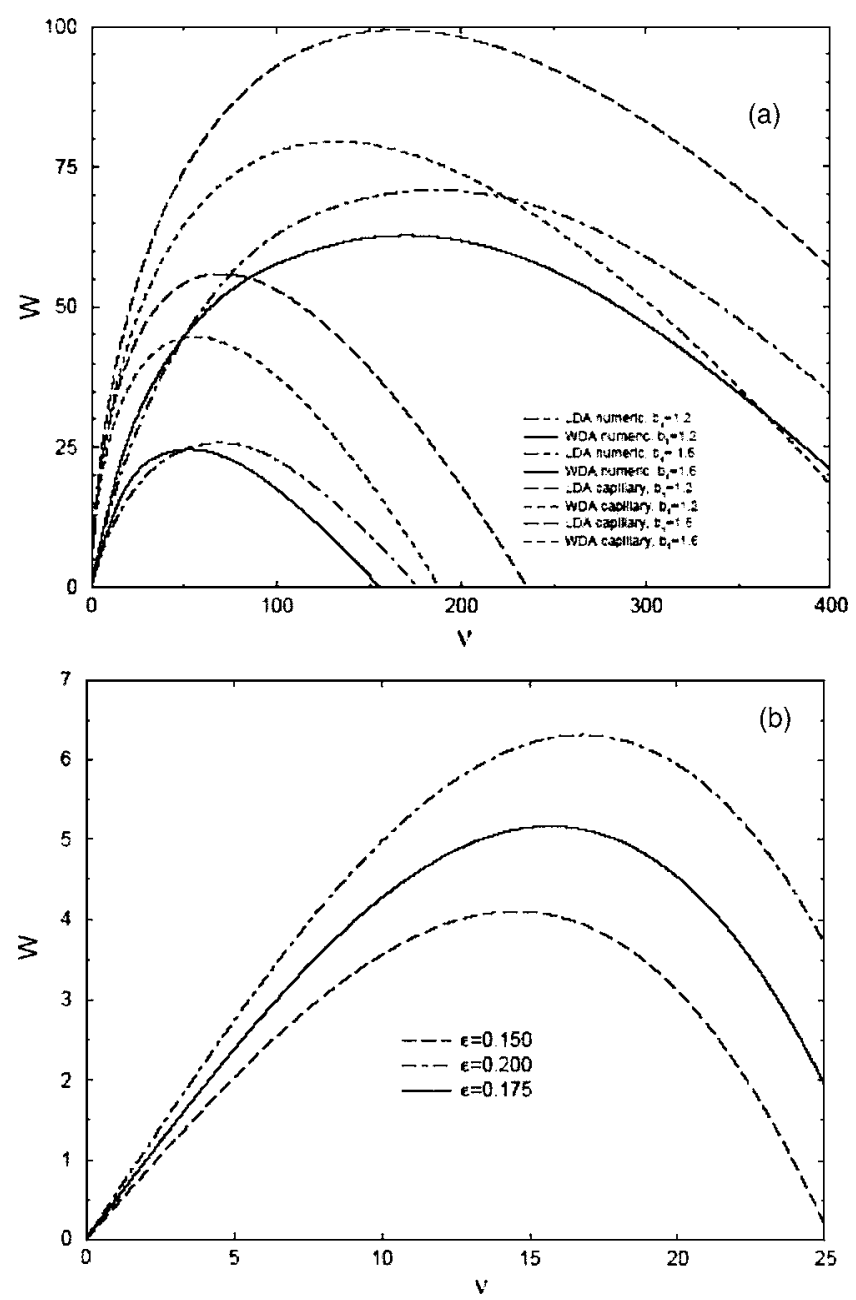

FIG. 6. (a) Formation free energy (in units of $k_{B} T$ ) of small homogeneous droplets as a function of the number of particles $\nu$ of the droplets at $T^{*}$ $=k_{B} T / \epsilon_{\mathrm{LJ}}=0.7$ and different values of supersaturation. (b) Formation free energy (in units of $k_{B} T$ ) of small homogeneous droplets in the near-threshold region as a function of the number of particles $\nu$ of the droplets at $T^{*}$ $=k_{B} T / \epsilon_{\mathrm{LJ}}=0.7$.

$$
W(\nu)=\int_{0}^{\nu} d \widetilde{\nu} b(\widetilde{\nu})-b_{1} \nu
$$

In homogeneous nucleation with $b_{1}<b_{\text {th }}$, the formation free energy is a nonmonotonic function of the droplet size and exhibits a maximum. This maximum corresponds to the nucleation barrier and the critical cluster.

Figure 6(a) depicts formation free energy curves in the case of high supersaturations where the differences between the classical theory and DFT are large. In general, the DFT predicts a lower height of nucleation barrier than the classical theory. Secondly DFT/WDA gives a lower barrier height than LDA. It is known that classical nucleation theory generally overestimates the nucleation rate by several orders of magnitude at high supersaturations compared to experiments. ${ }^{34}$ Similar situation could occur when comparing classical theory with our WDA results. Meanwhile the LDA results presented in Fig. 6(a) would cause almost 20 orders of magnitude difference in nucleation when compared with classical theory. As pointed out in the previous work ${ }^{1}$ LDA tends to overestimate the planar surface tensions compared with the more accurate WDA. Since this surface tension is used for classical calculations of formation free energy, the predicted difference in nucleation rate can be very large with the LDA. In this aspect, it is more desirable to use the more accurate WDA for homogeneous nucleation. To further describe kinetics of nucleation at some particular nucleation conditions, we need to analyze the shape of the curve in Fig. 6(a), including not just the height of nucleation barrier [maximum on the curve $W(\nu)]$ but also the location of the maximum (size of the critical cluster) and the half-width of the maximum. As shown in Fig. 6(a), WDA predicts a smaller critical size compared to LDA. The critical size predicted from the classical nucleation theory is much larger than that predicted from DFT. At low supersaturations both classical and DFT results are much closer. This is in part due to the fact that ${ }^{1,11}$ the Tolman length in the limit of the large droplets is very close to zero. As such, the classical nucleation theory can provide quite accurate description of the nucleation at low supersaturation.

We now analyze formation free energy in the nearthreshold region, where the chemical potential of vapor is very close to the spinodal value $\mu_{s 1}$. In this region, we can define an $\epsilon$ parameter via

$$
b_{1}=b_{\text {th }}(1-\epsilon) \text {, }
$$

where the barrierless nucleation occurs at $\epsilon=0$ and the nearthreshold region refers to $\epsilon \ll 1$. Parabolic approximation of Eq. (41) can be used for the chemical potential in this region. Integration of Eq. (41) gives

$$
W=\int_{0}^{\nu}\left(b_{s 1}-\frac{1}{2} A \widetilde{\nu}^{2}-b_{s 1}+\epsilon b_{s 1}\right) d \widetilde{\nu}=\epsilon b_{s 1} \nu-\frac{1}{6} A \nu^{3} .
$$

Critical droplets in the near-threshold region have a size of

$$
\nu_{c}=\left(\frac{2 \epsilon b_{s 1}}{A}\right)^{1 / 2}
$$

and half-width of the nucleation barrier is given by

$$
\Delta \nu_{c}=\left(\frac{2}{\epsilon b_{s 1} A}\right)^{1 / 4} \text {. }
$$

Substituting Eq. (46) into Eq. (45) gives

$$
\Delta W=\frac{2}{3} \sqrt{2} \frac{\epsilon^{3 / 2} b_{s 1}^{3 / 2}}{A^{1 / 2}}
$$

that is, the height of nucleation barrier. To satisfy parabolic approximation and to be able to use the classical theory for kinetics of nucleation, the condition ${ }^{32}$

$$
\frac{\Delta \nu_{c}}{\nu_{c}}=\left(\frac{A}{2 \epsilon^{3} b_{s 1}^{3}}\right)^{1 / 4} \ll 1
$$

should be met. For given $A$ and $b_{s 1}$ this means that $\epsilon$ should be larger than 0.15 . The condition of Eq. (49) also means that the dimensionless height of nucleation barrier described by Eq. (48) should be more than 2/3. Another condition that allows treating $\nu$ as continues variable in kinetics of nucleation is that ${ }^{32}$ 


$$
\Delta \nu_{c} \gg 1
$$

In our case it requires $\epsilon$ to be at least less than 0.20 . So we can identify the relatively small region of $0.15<\epsilon<0.20$ (for given temperature $\left.T^{*}=k_{B} T / \epsilon_{\mathrm{LJ}}=0.7\right)$ as the one where the simplified near-threshold description of homogeneous nucleation according to Eqs. (45)-(48) becomes possible. Figure 6(b) shows formation free energy curves for several values of supersaturation in the near-threshold region.

\section{HETEROGENEOUS NUCLEATION}

Again we use the radius of equimolar dividing surface to describe the size of droplets, including some modification for the case of heterogeneous nucleation. Equation (26) now becomes

$$
\begin{aligned}
4 \pi \int_{\tilde{R}_{n}}^{L} r^{2} \rho(r) d r= & 4 \pi R_{n}^{2} \Gamma_{32}+\frac{4 \pi}{3}\left(R_{e}^{3}-R_{n}^{3}\right) \rho_{2} \\
& +\frac{4 \pi}{3}\left(L^{3}-R_{e}^{3}\right) \rho_{1} .
\end{aligned}
$$

This equation involves three unknown quantities: radii of dividing surfaces $R_{e}$ and $R_{n}$, and the adsorption of liquid on the surface of the particle $\Gamma_{32}$. We used the same approach as in Ref. 1 and defined the dividing surface $R_{n}$ such that $\Gamma_{32}$ $=$ const $=\Gamma_{31 s}$, where $\Gamma_{31 s}$ is the adsorption of gas on the surface of the particle at the gas spinodal. Since bulk liquid cannot exist below the gas spinodal, the thickness of the liquid film becomes zero at this spinodal and Eq. (51) has the form

$$
\left.\int_{\tilde{R}_{n}}^{L} r^{2} \rho(r) d r\right|_{\mu=\mu_{s 2}}-\frac{L^{3}}{3} \rho_{2 s}=R_{n}^{2} \Gamma_{32}-\frac{R_{n}^{3}}{3} \rho_{1 s} .
$$

Another special case is the phase coexistence where liquid film becomes infinitely thick. In this case,

$$
\left.\int_{\tilde{R}_{n}}^{L} r^{2} \rho(r) d r\right|_{\mu=\mu_{\infty}}-\frac{L^{3}}{3} \rho_{2 \infty}=R_{n}^{2} \Gamma_{32}-\frac{R_{n}^{3}}{3} \rho_{2 \infty} .
$$

Solving the last two equations gives the solution for the two unknowns $R_{n}$ and $\Gamma_{32}$. So we have

$$
R_{n}^{3}=\frac{1}{\rho_{2 \infty}-\rho_{1 s}} \int_{0}^{L} r^{3}\left(\left.\frac{\partial \rho}{\partial r}\right|_{\mu=\mu_{\infty}}-\left.\frac{\partial \rho}{\partial r}\right|_{\mu=\mu_{s 1}}\right) d r .
$$

Recall that $z_{0} \equiv \widetilde{R}_{n}-R_{n}$. We found that $z_{0}$ increases as the particle size decreases. For instance, $z_{0}=2.35 d$ at $T^{*}$ $=k_{B} T / \epsilon_{\mathrm{LJ}}=0.7$ in the planar limit, while it becomes $z_{0}$ $=2.75 d$ for $\widetilde{R}_{n}=5.0 d$, and $z_{0}=3.31 d$ for microscopic size of $\widetilde{R}_{n}=3.5 \mathrm{~d}$. In Ref. 1 we used planar limiting value of $z_{0}$ to describe the position of the dividing surface for mesoscopic particles. This seems accurate since $z_{0}$ changes significantly only for microscopic particles. The adsorption $\Gamma_{32}$ can then be calculated for given $\widetilde{R}_{n}$. At $T^{*}=k_{B} T / \epsilon_{\mathrm{LJ}}=0.7, \quad \Gamma_{32}^{*}$ $=\Gamma_{32} \pi d^{2} / 6=0.77$ in the planar limit, but $\Gamma_{32}^{*}=0.52$ for $\widetilde{R}_{n}$ $=5 d$ and $\Gamma_{32}^{*}=0.43$ for $\widetilde{R}_{n}=3.5 d$. Thus, the adsorption decreases with decreasing particle size.
Once the radius of the dividing surface and the adsorption are known, we can then calculate the equimolar radius based on the density profiles for heterogeneous droplets as

$$
R_{e}^{3}=\frac{3}{\rho_{2}-\rho_{1}}\left(\int_{\tilde{R}_{n}}^{L} r^{2}\left(\rho(r)-\rho_{1}\right) d r+\frac{R_{n}^{3}}{3} \rho_{2}-R_{n}^{2} \Gamma_{32}\right) .
$$

This equimolar radius $R_{e}>R_{n}$ for any $\mu>\mu_{s 2}$.

\section{A. Chemical potential of the liquid condensate}

As mentioned previously, knowing the chemical potential of the liquid condensate as a function of droplet size is required to determine all necessary characteristics of nucleation. For heterogeneous nucleation on mesoscopic particles we obtained Eq. (39) in Ref. 1. However, the approximation used in Ref. 1, such as the use of first-order curvature correction and the planar term of disjoining pressure, is only valid for mesoscopic droplets. In the case of microscopic particles it is difficult to derive an analytical expression for the dependence of the chemical potential on the radius of the droplet. But this dependence can be obtained numerically based on DFT calculation. Towards this end, we first computed density profiles for given chemical potential and then the radius of the droplet.

The chemical potential of the liquid condensate as a function of equimolar radius is presented in Fig. 7(a) for $\widetilde{R}_{n}=5.0 d$, along with a plot of the analytical dependence Eq. (39) in Ref. 1 and chemical potential for homogeneous droplet. It can be seen that the chemical potential is a nonmonotonic function of the radius for heterogeneous nucleation but a monotonic function for homogeneous nucleation. The former result means that two sizes of equilibrium droplet can form on the foreign particle for $\mu_{\infty}<\mu<\mu_{\mathrm{th}}$. The smaller droplet represents a stable equilibrium solution, whereas the larger droplet represents an unstable critical droplet. The threshold value of chemical potential $\mu_{\text {th }}$ is difficult to calculate accurately due to the highly unstable nature of the density profile near the threshold. Figure 7(a) also shows that analytical Eq. (39) in Ref. 1 overestimates $\mu_{\text {th }}$ even for relatively large particles with radius of $\widetilde{R}_{n}=5.0 d$, indicating that Eq. (39) in Ref. 1 is not applicable for particles of smaller size. The deviation of Eq. (39) in Ref. 1 from the numerical results is due mainly to several approximations, including the dependence of the disjoining pressure on curvature (which we will discuss in the next section), stronger effect than the Tolman effect for the dependence of the surface tension on curvature, and dependences of $R_{n}$ and $\Gamma_{32}$ on the particle size.

Calculation of free energy of droplet formation requires not only the $R$ dependence of chemical potential but also the chemical potential as a function of the number of particles $\nu$ in the liquid condensate. In the case of heterogeneous nucleation this number is given by

$$
\nu=\frac{4 \pi}{3}\left(R_{e}^{3}-R_{n}^{3}\right) \rho_{2}+4 \pi R_{n}^{2} \Gamma_{32},
$$

where $\rho_{2}$ is the bulk density of liquid and $\Gamma_{32}$ is the adsorption which, according to our choice, is constant for $R>R_{n}$. 

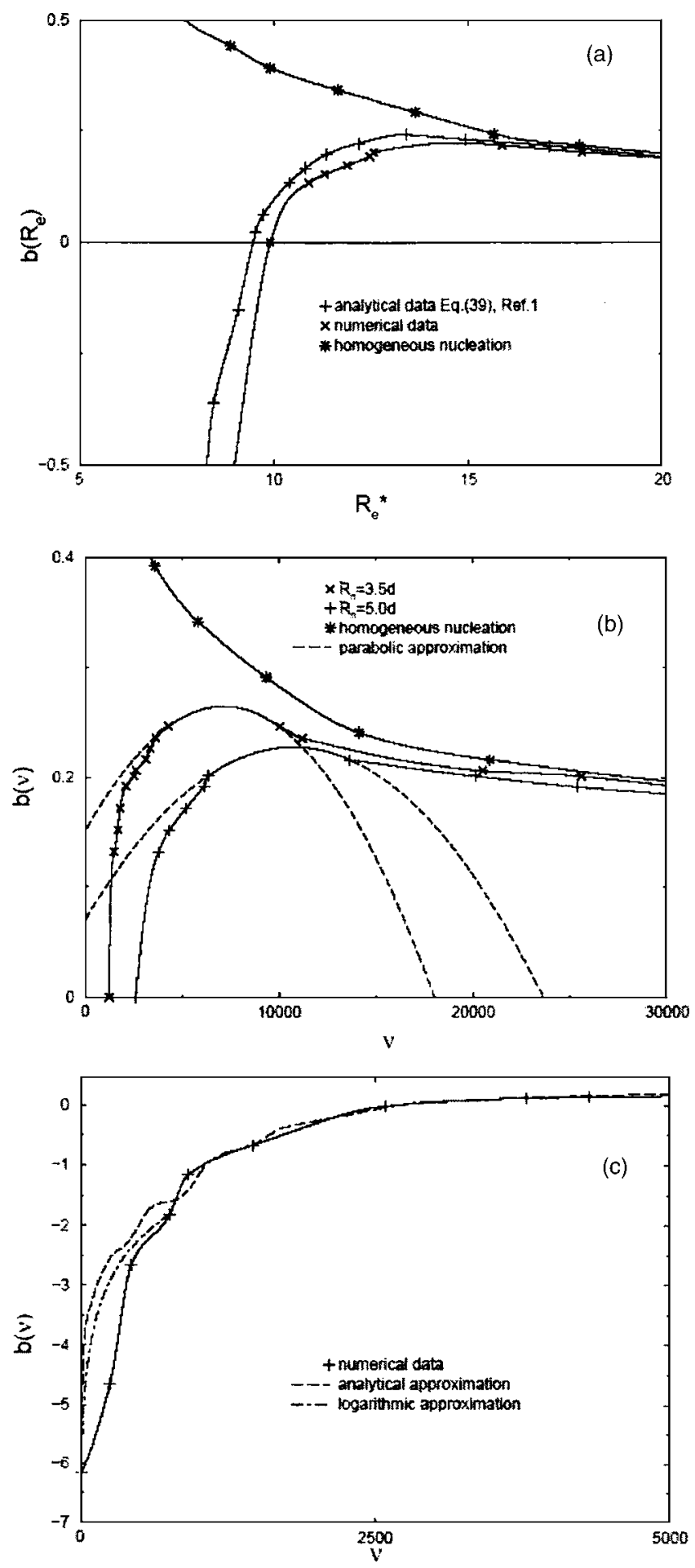

FIG. 7. (a) The dependence of the reduced chemical potential of the liquid condensate $b=\left(\mu-\mu_{\infty}\right) / k_{B} T$ on the equimolar radius $R_{e}^{*}=R_{e} / d$ of droplets formed on the foreign particle of radius $\widetilde{R}_{n}=5.0 d$ at $T^{*}=k_{B} T / \epsilon_{\mathrm{LJ}}=0.7$. (b) The dependence of the reduced chemical potential of the liquid condensate $b=\left(\mu-\mu_{\infty}\right) / k_{B} T$ on the number of particles $\nu$ of droplets with two different sizes of foreign particles at $T^{*}=k_{B} T / \epsilon_{\mathrm{LJ}}=0.7$. (c) The dependence of the reduced chemical potential of the liquid condensate $b=\left(\mu-\mu_{\infty}\right) / k_{B} T$ on the number of particles $\nu$ of droplets formed on the foreign particle of radius $\widetilde{R}_{n}=5.0 d$ at $T^{*}=k_{B} T / \epsilon_{\mathrm{LJ}}=0.7$.

For $R_{e}=R_{n}, h=0$ and $\Gamma_{32}=\Gamma_{31}$ at $\mu=\mu_{s 2}$. However, if $\mu$ $<\mu_{s 2}, \Gamma_{32}$ is not well defined and $\Gamma_{31}$ cannot be considered as constant. In fact, whenever $\mu<\mu_{s 2}$ liquid condensate does not exist in the form of the liquid film but in the form of adsorbed film on the surface of the solid particle. So

$$
\nu\left(\Gamma_{31}\right)=4 \pi R_{n}^{2} \Gamma_{31}=4 \pi \int_{0}^{L} r^{2}\left(\rho(r)-\rho_{1}\right) d r+\frac{4 \pi}{3} R_{n}^{3} \rho_{1} .
$$

Thus, for $\mu<\mu_{s 2}, \nu$ no longer depends on $R$ but on $\Gamma_{31}$ which changes from zero to $\Gamma_{32}$. Figure $7(\mathrm{~b})$ shows typical dependence of chemical potential of the liquid condensate on the number of particles in the liquid film with nucleation center of microscopic size. Again, the dependence is nonmonotonic.

In Ref. 1 we also discussed the behavior of chemical potential for $\mu<\mu_{s 2}$ and its relation to the law of adsorption. We showed there that the isotherm of adsorption $\left(\Gamma_{31}\right.$ vs $\left.p_{1}\right)$ is a stepwise monotonic function. The stepwise nature of this isotherm appears due to the oscillatory behavior of the density profile for the adsorbed film near the solid surface, which is slightly different from classical Henry's law of adsorption. Figure 7(c) depicts the reduced chemical potential $b$ vs $\nu$ for $\widetilde{R}_{n}=5.0 \mathrm{~d}$ for the case of adsorbed films. The figure also shows the classical logarithmic and analytical relation for mesoscopic particles. ${ }^{1}$ One can see that all the curves are close to each other. Weak oscillations do appear on the $b(\nu)$ curves, as shown before in Ref. 1. The positions of the oscillations as well as positions of the matching point between logarithmic and structured parts of the chemical potential curve are shifted due to microscopic-particle effects.

A key feature of the $b(\nu)$ curves [Fig. 7(b)] is the existence of a maximum $b_{m}=b_{\text {th }}$ at certain $\nu_{m}$. Since it is difficult to determine the threshold value of the chemical potential and $\nu_{m}$ with high accuracy due to strong instability of density profile, we estimated them by using a parabola relation [Eq. (56) in Ref. 1] fitting to the numeric data [Fig. 7(b)]. With the parabolic approximation in the near-threshold region, the numbers of molecules in equilibrium and critical droplets are given by

$$
\begin{aligned}
& \nu_{e}=\nu_{m}-\left(2 \epsilon b_{\mathrm{th}} / A\right)^{1 / 2}, \\
& \nu_{c}=\nu_{m}+\left(2 \epsilon b_{\mathrm{th}} / A\right)^{1 / 2},
\end{aligned}
$$

where $b_{\text {th }}, \nu_{m}$, and $A=\left|d^{2} b(\nu) / d \nu^{2}\right| \nu_{m}$ are fitted to the chemical potential curves. The fitting values are $b_{\text {th }}=0.2283, \nu_{m}$ $=10750$, and $A=2.745 \times 10^{-9}$ for $\widetilde{R}_{n}=5.0 d$ and $b_{\mathrm{th}}=0.2650$, $\nu_{m}=7154$, and $A=4.492 \times 10^{-9}$ for $\widetilde{R}_{n}=3.5 \mathrm{~d}$.

\section{B. Discussion of disjoining pressure}

The discrepancies in predictions of analytical equation for the chemical potential of the liquid condensate [Eq. (39) in Ref. 1] and numerical results of the present work [Fig. $7(\mathrm{a})]$ are likely due to the difference in the disjoining pressure for the film on the spherical microscopic particle from the one on the planar substrate. In addition to volumelike disjoining pressure contribution, Eq. (39) in Ref. 1 also includes surface tension contributions. In our discussion of homogeneous droplets we showed that these surfacelike contributions are significantly different from the ones predicted from pure Tolman correction as Eq. (39) of Ref. 1. This is 

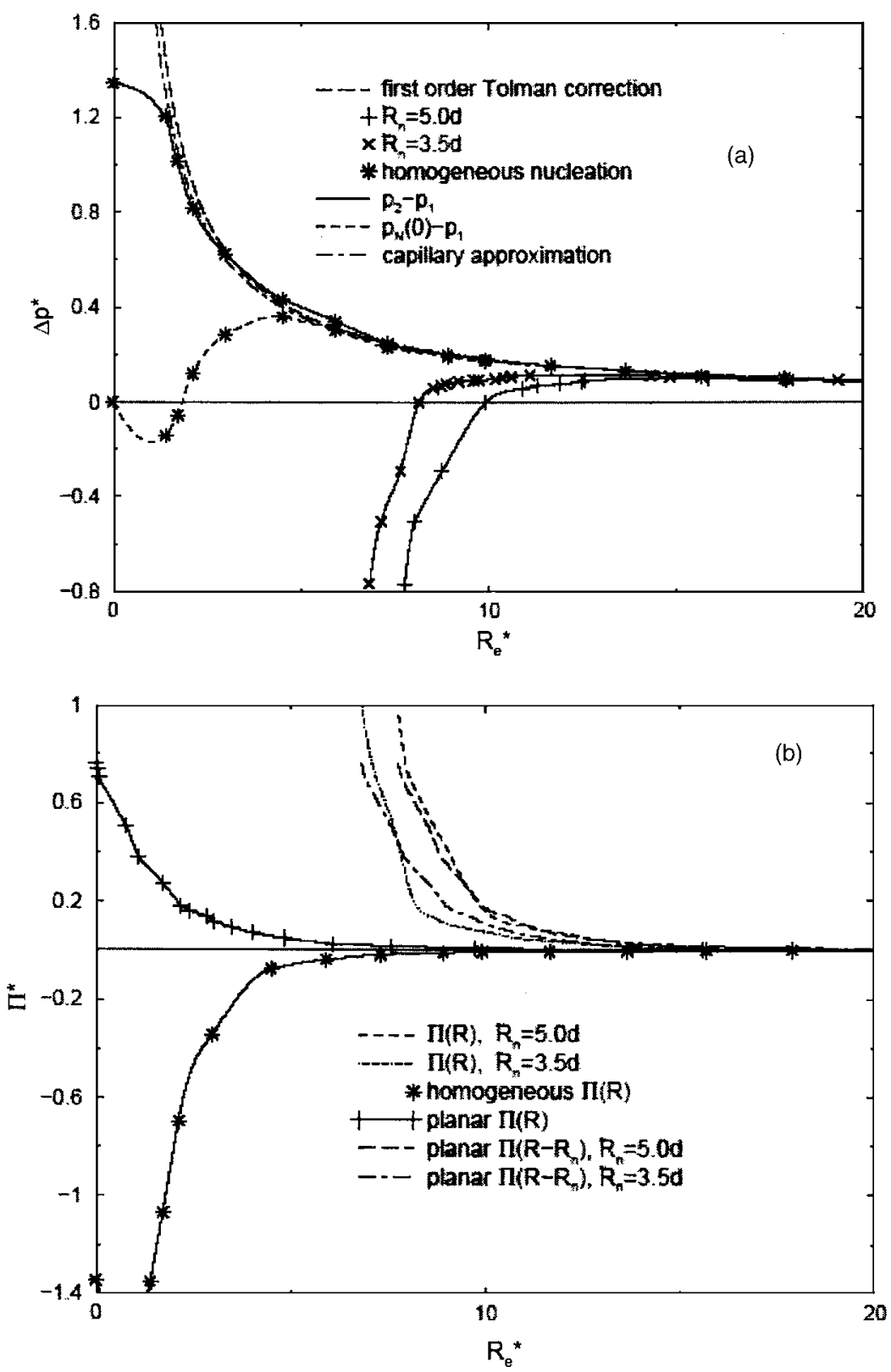

FIG. 8. (a) The reduced Laplacian pressure difference $\Delta p^{*}=\Delta p \pi d^{3} / 6 k_{B} T$ as a function of the equimolar radius $R_{e}^{*}=R_{e} / d$ of the droplets of different types at $T^{*}$ $=k_{B} T / \epsilon_{\mathrm{LJ}}=0.7$. (b) The reduced disjoining pressure $\left(\Pi^{*}=\Pi \pi d^{3} / 6 k_{B} T\right)$ of different droplets vs the equimolar dividing surface $\left(R_{e}^{*}=R_{e} / d\right)$ at $T^{*}=k_{B} T / \epsilon_{\mathrm{LJ}}=0.7$. another reason why Eq. (39) overestimates the threshold value of chemical potential for microscopic droplets.

To further explain the discrepancies we started by plotting the Laplacian pressure difference for bulk phases as a function of the equimolar radius of the critical droplet. Figure 8(a) depicts this pressure difference for several cases. For small homogeneous droplets, the pressure difference between bulk phases differs from the one predicted by classical capillary approximation $\left(p_{2}-p_{1}=2 \sigma_{21 \infty} / R\right)$, and the one predicted by first-order Tolman curvature correction theory $\left[p_{2}\right.$ $\left.-p_{1}=2 \sigma_{21 \infty} / R\left(1-\delta_{\infty} / R\right)\right]$. In the Laplace equation (8) both the surface tension term and the disjoining pressure term are strongly dependent on the curvature. As a result the finite value of the pressure difference takes place in liquid spinodal, while classical theory predicts this value to grow infinitely as radius $R$ approaches to zero. Meanwhile, we can plot the difference between normal components of the pressure tensor inside of the droplet and outside of the droplet [Eq. (10)]. This curve demonstrates a nonmonotonic behav- ior. Such pressure differences only include surfacelike contributions. The difference between the two curves is the negative disjoining pressure of homogeneous droplet. On the other hand when the radius of the droplet is large enough (larger than $15 d$ in our graph) all the curves are in agreement with the classical approximation.

Figure 8(a) also shows the bulk pressure difference in the case of heterogeneous nucleation on microscopic particles, which behaves according to the general Laplace equation (3). In such a case the pressure difference is a nonmonotonic function of the droplet size, resulting in the appearance of the stable equilibrium clusters on the surface of microscopic foreign particle. The Laplace equation (3) was introduced so that the surface contribution in this equation reflects the dependence of the surface tension on the external radius of the droplet, but it is independent of the foreign particle inside of the droplet. To calculate the disjoining pressure one can subtract homogeneous surfacelike contribution from heterogeneous bulk pressure difference. 
The disjoining pressure as a function of the droplet equimolar radius is plotted in Fig. 8(b), given the size of foreign particles $\widetilde{R}_{n}=3.5 d$ and $\widetilde{R}_{n}=5.0 d$. It can be seen that the negative homogeneouslike contribution to disjoining pressure is only significant if the external radius of the droplet is less than $2.5 d$. In the case of microscopic particles considered here the influence of homogeneouslike contribution to disjoining pressure is not strong. Here, we do not consider microscopic particles with size less than $3 d$ since nucleation would be more like binary nucleation which is beyond the framework of the present study.

In the case of nucleation on mesoscopic particles considered in Ref. 1 we replaced the disjoining pressure of the film on the spherical particle by the disjoining pressure of the planar film with thickness $h=R-R_{n}$. Figure 8 (b) also displays this planar disjoining pressure as function of $R$ for both particle sizes considered here. The particle size $\widetilde{R}_{n}=5 d$ represents a borderline case between the mesoscopic and microscopic particles. It is acceptable to use the planar approximation to the disjoining pressure ${ }^{1}$ for sizes larger than $5 d$. One of the reasons is that in the present model the potential of the foreign particle depends only on the distance from the particle surface. In addition, the homogeneouslike contribution to the disjoining pressure is almost negligible for larger particles. Hence, the differences between the behavior of the chemical potential from Eq. (39) of Ref. 1 and the one shown in Fig. 7(a) are not due to the disjoining pressure but rather the surfacelike contributions. Figure 8(a) shows that these contributions cause the behavior different from the Tolmantype behavior even at $R=5 d$. This problem should not occur for $\widetilde{R}_{n}$ larger than $5 d$ in Ref. 1.

From Fig. 8(b). one can see that for $\widetilde{R}_{n}=3.5 d$ the disjoining pressure is different from the planar one. For much thicker films it is lower than the planar value. This result is consistent with the previous study of films on the spherical core particles, ${ }^{35}$ even though a different Yukawa-type particle potential and a slightly different definition of the disjoining pressure were used in that work. It is more difficult to compare planar and spherical disjoining pressures for thinner films, since not only the disjoining pressure itself but also the position of the dividing surface $R_{n}$ depend on the particle size.

All in all, we conclude that the analytical method developed in Ref. 1 works fairly well for $\widetilde{R}_{n}>5 d$. It gives reliable results of chemical potential and disjoining pressure. Numerical method is required for the size range within $3 d$ $<\widetilde{R}_{n}<5 d$.

\section{Free energy of droplet formation and nucleation barrier height}

Once the chemical potential of the liquid condensate as function of the number of molecules $b(\nu)$ is known, we can calculate the free energy of droplet formation based on Eqs. (42) and (43). Here the boundary condition $W(0)=0$ in Eq. (43) means that the free energy of droplet formation is zero for a bare solid particle.

One can substitute $b(\widetilde{\nu})$ into Eq. (43) to obtain Eq. (69) of
Ref. 1, which is still valid with account of small compressibility. However, since we do not have the analytical relation $b(R)$ as we had in Ref. 1 for mesoscopic foreign particles, we need to perform numerical integration of the $b(\nu)$ presented in Fig. 7. The resulting free energy of droplet formation $W(\nu)$ for given $b_{1}$ and two radii $\widetilde{R}_{n}=3.5 d$ and $\widetilde{R}_{n}=5.0 d$, respectively, are shown in Fig. 9. As expected, all the $W(\nu)$ curves exhibit an $S$-like feature, a signature of the heterogeneous nucleation. The minima on these curves correspond to the equilibrium clusters of size $\nu_{e}$, for which microscopic solid particle is covered by a stable liquid film with finite thickness. The value $W_{e}=W\left(\nu_{e}\right)$ is negative, indicating the spontaneous formation of equilibrium cluster. The absolute value of $W_{e}$ depends on the accuracy of numerical integration of $b(\nu)$ for small $\nu$, where less points on the $b(\nu)$ curve are available. Note that $W_{e}$ itself is not essential to the kinetics of nucleation, because it is only a reference point (constant of integration) for evaluating nucleation barrier. The minima become less deep as the size of the foreign particle decreases. It disappears in the limiting case of homogeneous nucleation, namely, at $\nu=0$. The maxima on the $W(\nu)$ curves at $\nu_{c}$ correspond to the critical cluster and give the peak of the barrier.

The height of the nucleation barrier $\Delta W \equiv W\left(\nu_{c}\right)$ $-W\left(\nu_{e}\right)$ should be less than several tens of $k_{B} T$ to give measurable nucleation rates. This case occurs typically at the near-threshold region as the chemical potential of vapor $b_{1} \rightarrow b_{\text {th }}$ from below. In this case both $\nu_{e}$ and $\nu_{c}$ are approaching to the same value $\nu_{m}$ for the maximum on $b(\nu)$ curve. The $\nu_{m}$ value depends on the size of the foreign particle, approaching to zero if nucleation is homogeneous, but it does not depend on the supersaturation of vapor.

It had been shown ${ }^{7}$ that in the near-threshold region, where the parabolic approximation for the chemical potential $b(\nu)$ is valid, the nucleation barrier can be written as

$$
\Delta W=\frac{4}{3} \epsilon^{3 / 2} b_{\mathrm{th}}^{3 / 2}\left(\frac{2}{A}\right)^{1 / 2},
$$

and the half-widths $\Delta \nu_{e}$ and $\Delta \nu_{c}$ of the minimum and maximum on the $W(\nu)$ curve can be given by

$$
\Delta \nu_{e}=\Delta \nu_{c}=\left(\frac{2}{\epsilon b_{\mathrm{th}} A}\right)^{1 / 4}
$$

Equation (59) can be used to determine the nucleation barrier height once $b_{\text {th }}$ and $A$ are obtained by fitting to the $b(\nu)$ curves. Since this equation is applicable only to the near-threshold region, we need to specify certain limits for $\epsilon$ parameter. By inspecting $b(\nu)$ curves in Fig. 7(b) we found that $\epsilon$ parameter should be at least 0.07 or less in order to use the parabolic approximation. The value 0.07 is about the same as the upper limit we derived for the $\epsilon$ in Ref. 1. That upper limit is also regulated by the condition similar to Eq. (50). For the case of heterogeneous nucleation, this condition becomes 

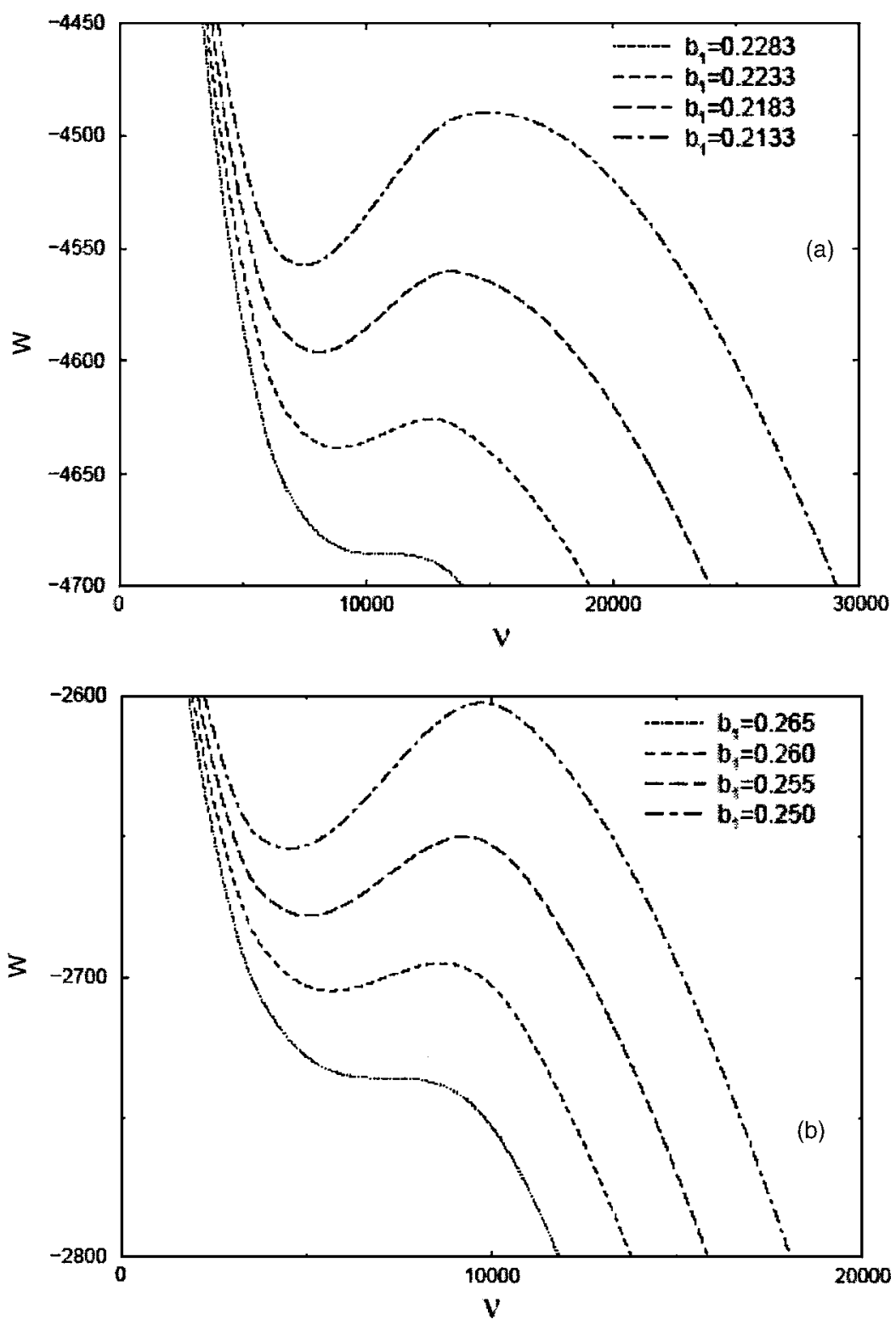

FIG. 9. (a) Formation free energy (in units of $k_{B} T$ ) of droplets formed on the foreign particle of size $\widetilde{R}_{n}$ $=5.0 \mathrm{~d}$ as function of the number of particles $\nu$ of the droplet at $T^{*}=k_{B} T / \epsilon_{\mathrm{LJ}}=0.7$ and different supersaturations. (b) Formation free energy (in units of $k_{B} T$ ) of droplets formed on the foreign particle of size $\widetilde{R}_{n}$ $=3.5 \mathrm{~d}$ as function of the number of particles $\nu$ of the droplet at $T^{*}=k_{B} T / \epsilon_{\mathrm{LJ}}=0.7$ and different supersaturations.

$$
\begin{aligned}
& \Delta \nu_{e} \gg 1, \\
& \Delta \nu_{c} \gg 1,
\end{aligned}
$$

which allow to treat the number of particles as a continuous variable in kinetic theory of nucleation. For given $\widetilde{R}_{n}$ these conditions are still working very well. The low limit of $\epsilon$ parameter is controlled by the validity condition for parabolic approximation ${ }^{7,8}$ similar to the condition of Eq. (49). For heterogeneous case this condition ${ }^{7}$ requires the height of the barrier to be larger than $2 / 3 \sqrt{3}$ and the $\epsilon$ parameter to be at least larger than 0.005 for the value of $\widetilde{R}_{n}$ considered here.

In summary, the near-threshold region predicted is nearly the same as in Ref. 1 . The $\epsilon$ parameter can vary from 0.006-0.007 to about 0.05-0.06. In the latter region one can use Eqs. (59) and (60) to evaluate the barrier height and width. The height ranges from about $2 k_{B} T$ to few tens of $k_{B} T$. For other values of $\epsilon$ parameter only numerical results (from formation free energy curves in Fig. 9) can be used.

\section{CONCLUSIONS}

In this work we have studied two major subjects. The first is the heterogeneous nucleation on microscopic wettable particles and the second is the homogeneous nucleation at high supersaturation. The theoretical approach undertaken is an extension of the hybrid thermodynamic/DFT method proposed previously to deal with heterogeneous nucleation on mesoscopic wettable particles. In particular, we extended the thermodynamic part of the theory in order to describe the disjoining pressure and the surface tension of very small droplets.

The advantage of the present theoretical approach over classical one is that it can account for the curvature dependence of the surface tension as well as compressibility corrections and effects of the disjoining pressure. We studied these corrections based on DFT calculation for LennardJones fluid. Even though our conclusions are based on this particular model, the physical insights established from this study are more general and can be valid for many other types of model fluids. For both nucleation problems considered 
here, the parameters $d / R$ and $d / R_{n}$ are no longer small enough as in the case of mesoscopic nucleation center. The absence of these small parameters renders analytical approach invalid and numerical calculations are required to compare with previous results. Some general conclusions obtained from this study are given below.

We examined different contributions to the disjoining pressure of LJ liquid film on the surface of the spherical particle. We showed that van der Waals interactions provide negative contribution to disjoining pressure. This contribution which depends on both thickness of the liquid film and radius of the foreign particle can be accounted for by means of the power-law approximation if the thickness of the film exceeds several molecular diameters. The parameters involved in the power-law approximation are related to the parameters of intermolecular potential. The van der Waals contribution dominates the disjoining pressure of homogeneous droplets. On the other hand, the presence of the wettable nucleation center provides positive contribution to the disjoining pressure. This contribution is predominant for mesoscopic particles considered in Ref. 1 as well as for microscopic particles considered here, resulting in the monotonic disjoining pressure as function of the film thickness. We also confirmed that the planar approximation we used previously for mesoscopic particles, including the power-law approximation for thick films and exponential approximation for thin films, is accurate.

We studied curvature dependence of the surface tension. In the case of homogeneous droplets we compared the results from both LDA and WDA within the framework of DFT and found that they both lead to similar predictions. The surface tension is strongly dependent on the curvature of the droplet, particularly when droplets are smaller than several molecular diameters and the Tolman length cannot be treated as a constant. The importance regarding definitions of the surface tension for different dividing surfaces is discussed. We also discussed the difference between the physical natures of volumelike (the disjoining pressure) and surfacelike (the surface tension) contributions to thermodynamic potentials of the system. We found that the surfacelike contributions are mostly responsible for deviations from the predictions of classical nucleation theory for homogeneous nucleation and heterogeneous nucleation on macroscopic particles. ${ }^{7}$

We examined the dependence of the chemical potential of the liquid condensate on the size of the droplet. We confirmed that this dependence is a monotonic function in the case of homogeneous nucleation, regardless of LDA and WDA. However, the chemical potential dependence shows significant deviations from the prediction of the classical capillary approximation. If the size of the droplet is less than several molecular diameters the chemical potential is much lower compared to classical values. In particular, a threshold value of chemical potential exists, above which the nucleation becomes barrierless. In the case of heterogeneous nucleation on microscopic foreign particles the chemical potential of the liquid condensate is a nonmonotonic function of the droplet size. The maximum chemical potential has also the significance of the threshold value. Numerical calculation is required to obtain accurate value of the threshold. Our numerical calculation also confirmed that the analytical approximation used previously works well for mesoscopic particles.

We obtained several key quantities relevant to kinetics of nucleation, such as the free energy of droplet formation and the barrier height to nucleation. We confirmed that in the case of homogeneous nucleation both LDA and WDA give a lower barrier at high supersaturation compared to the prediction of classical nucleation theory. This lowering of the barrier is due to the overlapping of the surface layer of the small droplet and the finite value of the threshold chemical potential. In the case of heterogeneous nucleation on microscopic particles, the formation free energy as a function of droplet size has a minimum which represents a stable equilibrium cluster formed on the surface of the foreign particle, as well as a maximum which represents the unstable critical cluster. The height difference between these two extreme points corresponds to the nucleation barrier. Note that the nucleation rate depends exponentially on the height of nucleation barrier and is also inversely proportional to the barrier width [e.g., Eqs. (83)-(85) in Ref. 1 for heterogeneous nucleation]. We also showed that certain simplifications can be made in calculating parameters important to the kinetics of nucleation near the threshold. The results can be used as input parameters to compute rates of nucleation at various conditions. ${ }^{33}$

\section{ACKNOWLEDGMENTS}

This work is supported by grants from the DOE (DEFG02-04ER46164), NSF (CHE), and the Nebraska Research Initiative, and by the John Simon Guggenheim Foundation and the Research Computing Facility at University of Nebraska-Lincoln.

${ }^{1}$ T. V. Bykov and X. C. Zeng, J. Chem. Phys. 117, 1851 (2002).

${ }^{2}$ R. Becker and W. Doring, Ann. Phys. 24, 719 (1935).

${ }^{3}$ R. McGraw and A. Laaksonen, Phys. Rev. Lett. 76, 2754 (1996).

${ }^{4}$ V. Talanquer, J. Chem. Phys. 106, 9957 (1997).

${ }^{5}$ D. Reguera and H. Reiss, Phys. Rev. Lett. 93, 165701 (2004).

${ }^{6}$ D. Kashchiev, J. Chem. Phys. 118, 1837 (2003).

${ }^{7}$ F. M. Kuni, A. K. Shchekin, A. I. Rusanov, and B. Widom, Adv. Colloid Interface Sci. 65, 71 (1996).

${ }^{8}$ F. M. Kuni, A. K. Shchekin, and A. P. Grinin, Usp. Fiz. Nauk 171, 345 (2001).

${ }^{9}$ A. K. Shchekin, D. V. Tatianenko, and F. M. Kuni, in Nucleation Theory and Applications, edited by J. W. P. Schmelzer, G. Ropke, and V. B. Priezzhev (JINR, Dubna, 1999), p. 320.

${ }^{10}$ T. V. Bykov and X. C. Zeng, J. Phys. Chem. B 105, 11586 (2001).

${ }^{11}$ Y. A. Lei, T. V. Bykov, S. Yoo, and X. C. Zeng, J. Am. Chem. Soc. 127, 15346 (2005).

${ }^{12}$ K. Koga, X. C. Zeng, and A. K. Shchekin, J. Chem. Phys. 109, 4063 (1998).

${ }^{13}$ A. I. Rusanov, Phasengleichgewichte und Grenzflachenerscheinungen (Akademie, Berlin, 1978).

${ }^{14}$ T. V. Bykov and A. K. Shchekin, Colloid J. 61, 144 (1999).

${ }^{15}$ T. V. Bykov and X. C. Zeng, J. Chem. Phys. 111, 10602 (1999).

${ }^{16}$ I. Hadjiagapiou, J. Phys.: Condens. Matter 6, 5303 (1994).

${ }^{17}$ R. Tsekov, K. W. Stockelhunber, and B. V. Toshev, Langmuir 16, 3502 (2000).

${ }^{18}$ P. Tarazona, Phys. Rev. A 31, 2672 (1985).

${ }^{19}$ P. Tarazona, U. M. Marconi, and R. Evans, Mol. Phys. 60, 573 (1987).

${ }^{20}$ T. F. Meister and D. M. Kroll, Phys. Rev. A 31, 4055 (1985).

${ }^{21}$ J. R. Henderson, P. Tarazona, F. van Swol, and E. Velasco, J. Chem. Phys. 96, 4633 (1992).

${ }^{22}$ S. Dhawan, M. E. Reimel, L. E. Scriven, and H. T. Davis, J. Chem. Phys. 94, 4479 (1991). 
${ }^{23}$ X. C. Zeng and D. W. Oxtoby, J. Chem. Phys. 94, 4472 (1991).

${ }^{24}$ B. V. Derjaguin, N. V. Churaev, and V. M. Muller, Surface forces (Consultant Bureau, New York, 1987).

${ }^{25}$ C. Hamaker, Physica (Amsterdam) 1058 (1937).

${ }^{26}$ T. Getta and S. Dietrich, Phys. Rev. E 57, 655 (1998).

${ }^{27}$ R. J. F. Leote de Carvalho, R. Evans, D. C. Hoyle, and J. R. Henderson, J. Phys.: Condens. Matter 6, 9275 (1994).

${ }^{28}$ N. E. Carnahan and K. E. Starling, J. Chem. Phys. 51, 635 (1969).

${ }^{29}$ A. H. Falls, L. E. Scriven, and H. T. Davis, J. Chem. Phys. 75, 3986 (1981).
${ }^{30}$ D. H. Rasmussen, J. Cryst. Growth 56, 45 (1982).

${ }^{31}$ B. N. Hale, Phys. Rev. A 33, 4156 (1986).

${ }^{32}$ F. M. Kuni and A. P. Grinin, Vestn. Leningr. Univ., Ser. 4: Fiz., Khim. 22, 10 (1982)

${ }^{33}$ F. M. Kuni, A. P. Grinin, and V. B. Kurasov, in Nucleation Theory and Applications, edited by J. W. P. Schmelzer, G. Ropke, and V. B. Priezzhev (JINR, Dubna, 1999), p. 120.

${ }^{34}$ Y. Viisanen and R. Strey, J. Chem. Phys. 101, 7835 (1994).

${ }^{35}$ I. Napari and A. Laaksonen, J. Chem. Phys. 119, 10363 (2003). 